\section{Human exposure to mercury and its hematological effects: a systematic review}

\author{
Exposição humana ao mercúrio e seus efeitos \\ hematológicos: uma revisão sistemática
}

\section{Exposición humana al mercurio y sus efectos hematológicos: una revisión sistemática}

\author{
Angélica dos Santos Vianna 1 \\ Elisabete Pedra de Matos 2 \\ Iracina Maura de Jesus ${ }^{3}$ \\ Carmen Ildes Rodrigues Fróes Asmus 1,4 \\ Volney de Magalhães Câmara 1,4
}

\begin{abstract}
Mercury is a metal found in the environment from natural and anthropogenic sources. It is highly toxic to ecosystems and living beings. Most human exposures come from ingestion of contaminated seafood, outgassing from dental amalgam or occupational exposure (e.g. gold mining), among other cases. Large populations are exposed to mercury, making it a very important issue from the public health perspective. Adverse health effects are commonly seen in the nervous system, but every organ is a potential target, such as the bone marrow. The main goal of this study was to assess the available evidence on human exposure to mercury and its hematological effects. A search strategy was constructed, including key terms ( $\mathrm{MeSH}$, text word and equivalents) for querying 2 repositories of master dissertation and $\mathrm{PhD}$ thesis (Fiocruz/ARCA and University of São Paulo) and 4 different electronic databases: BVS/ LILACS, MEDLINE/PubMed, Scopus and TOXLINE/NIH, for articles published from 1950 to February 2018. There was no language restriction and a tool (EPHPP) was used to assess the quality of included studies. According to pre-established criteria, 80 studies were retrieved, all of them observational ( 48 case reports, 24 cross-sectional, 6 case series and 2 cohorts), comprising 9,284 people. Despite the fact that most exposed ones (6,012) had normal blood cell count and mercury hematological effects did not seem very usual $(1,914$ cases: 14 severe and 29 deaths), three studies reported association ( $\beta)$ for anemia, lymphopenia, neutrophilia and basophilia. We concluded that the gathered information pointed to mercury hematotoxic effects, some of them may be serious and even fatal.
\end{abstract}

Mercury Poisoning; Heavy Metal Poisoning; Mercury; Blood Cell Count

\section{Correspondence}

A. S. Vianna

Instituto de Estudos de Saúde Coletiva, Universidade Federal do Rio de Janeiro.

Av. Horácio Macedo s/n, Rio de Janeiro, RJ 21941-598, Brasil. angelica@iesc.ufrj.br

1 Instituto de Estudos de Saúde Coletiva, Universidade Federal do Rio de Janeiro, Rio de Janeiro, Brasil.

2 Pesquisadora Independente, Rio de Janeiro, Brasil.

3 Instituto Evandro Chagas, Ananindeua, Brasil.

4 Faculdade de Medicina, Universidade Federal do Rio de

Janeiro, Rio de Janeiro, Brasil. 


\section{Introduction}

Mercury is a heavy metal considered as the most toxic non-radioactive element in the world. It is ubiquitous, indestructible and exists in three forms in nature: inorganic, metallic and organic 1 . It is released to the atmosphere from four different sources: (i) primary natural (e.g. volcanic and geothermal activities), responsible for 10\%; (ii) primary anthropogenic (e.g. mining and fossil fuel extraction, including oil, gas and coal); (iii) secondary anthropogenic (mercury-dependent artisanal and small scale gold mining sector [ASGM], several industrial processes including chlor-alkali industry), both anthropogenic responsible for 30\%; and (iv) remobilization and re-emissions (wildfires, forest clearing, biomass burning), responsible for $60 \% 2$. Because it is a widespread environmental toxicant, humans are unable to avoid exposure to its forms 3 and the main sources are: fish and shellfish consumption, outgassing from dental amalgam, vaccines containing thiomersal and occupational exposure (agricultural products, industry and gold mining) ${ }^{4}$. Specifically in relation to the latter, ASGM is considered the number one anthropogenic mercury pollutant in the world, responsible for $37 \%$ (410 to 1,400 tones/year) of its emissions to air and water worldwide. It poses a risk not only to miners, estimated at 10 to 19 million workers, of which 5 million are women and children, in more than 70 countries, but also to the environment and general population by water and air 2,5 . Such large variation of human exposure to mercury makes it a very important issue from the public health perspective 6,7 .

All forms of mercury could poison cellular function by altering the tertiary and quaternary structure of proteins and membrane permeability due to its affinity for sulfhydryl and selenohydryl groups. As a consequence it can potentially impair function of any organ 8,9 . Its adverse effects on human health may induce over 250 symptoms. The main ones are from nervous, renal, cardiovascular, respiratory systems, and skin, but any organ may be a target, such as the bone marrow 9 . The hematological system, due to its intense cellular proliferation, is quite sensitive to the action of a variety of substances, such as benzene 10. However, there is sparse information and research about mercury's hematotoxicity on humans, despite its wide exposure 11. Most of them come from occupational settings 12,13 , in vitro 14,15 and animals studies 16,17 .

The aim of this systematic review was to assess the available evidence on human exposure to mercury and its hematological effects.

\section{Methods}

This systematic review followed the precepts established by the PRISMA model 18 and had a PROSPERO register: CRD42018086389.

\section{Data sources, search strategy and study selection}

The selection criteria were based on PICOS' acronymous 19: "Does human exposure to mercury lead to hematological effects?” and included all studies (except textbook, author's opinion and review) regarding human exposure to mercury and hematological effects, published between 1950 and February 2018. Hematological effects were considered as any blood cell alteration concerning number 20 and the normal values of mercury on biological matrices were those presented by the authors.

We developed a search strategy including key terms (MeSH, text word and equivalents) for querying four different electronic databases (BVS/LILACS; MEDLINE/PubMed; Scopus; and TOXLINE/ $\mathrm{NIH}$ ) and two Master dissertation/PhD thesis databases (Fiocruz/ARCA and University of São Paulo). There were four search strategies containing the descriptors according to database and repositories. For BVS/LILACS: "mercúrio” AND “anemia” OR "leucopenia” OR "basopenia” OR "eosinopenia” OR “neutropenia” OR "linfopenia” OR "monocitopenia” OR "trombocitopenia” OR "policitemia” OR "leucocitose” OR "basofilia” OR “eosinofilia” OR "neutrofilia” OR "linfocitose” OR "monocitose” OR "trombocitose” OR "hemograma completo”. For MEDLINE/PubMed: "anemia” OR "leukopenia” OR "thrombocytopenia” OR "eosinopenia” OR "basopenia” OR "monocytopenia” OR "polycythemia” OR “leukocytosis” OR "thrombocytosis” OR “eosinophilia” OR “basophilia” OR "neutrophilia” OR 
"monocytosis" OR "blood cell count" AND "mercury". For TOXLINE/NIH, the search was made in a binary way: anemia and mercury/leukopenia and mercury. For the two repositories: "mercúrio" and "efeitos hematológicos". Reference lists were also searched for relevant studies. No restrictions were applied concerning language and translation was done whenever necessary. Both authors (A.S.V. and E.P.M.) followed the same schedule independently: first they reviewed the title, then the available abstract, soon after the analysis of full text, and finally the search for reference. Any discrepancy in the search results not solved between A.S.V. and E.P.M. was planned to be discussed with a third author (C.I.R.F.A.). In order of priority, we excluded: non-human studies; without mercury exposure; lacking hematological effect; textbook, author's opinion and review papers (type of study); and those published before 1950. To determine agreement between the two raters, Cohen's kappa statistic was used for each step.

\section{Data extraction}

The extraction process was also done independently and included: author, year, place, journal, data base, type of study, substance, exposure (local and duration), population (number, exposed versus non exposed, age, sex), hematological outcome (primary or secondary), death, blood cell count, bone marrow biopsy, mercury (sample, level, method) and statistical analysis. Once more, Cohen's kappa statistic was used to evaluate an inter-rater agreement.

\section{Study quality assessment}

To assess the quality/bias risk of the selected studies, we chose a tool known as Effective Public Health Practice Project (EPHPP) 21. This quality assessment tool for quantitative studies has eight components ratings: (a) selection bias; (b) study design; (c) confounders; (d) blinding; (e) data collection methods; (f) withdrawals and drop-outs; (g) intervention integrity; and (h) analyses. Items from "a" to "f" are rated as strong (1), moderate (2) or weak (3). There is a dictionary to correctly rate each section. These six items are included in global rating, ranked as follows: strong must not have no weak ratings; moderate may have one weak rating, and weak may have two or more weak ratings. At the end there is an item for discrepancy between both reviewers that indicate the reason for discrepancy: oversight, differences in interpretation of criteria and differences in interpretation of study. That will lead to a final decision of both reviewers.

The results were summarized in a descriptive manner for occupational and non-occupational exposure data, due to toxicological differences between them.

\section{Results}

The search yielded 1,297 citations as of February 14th 2018, 323 from BVS/LILACS, 142 from MEDLINE/PubMed, 525 from Scopus, 110 from TOXLINE/NIH, 197 from Fiocruz/ARCA and none from the University of São Paulo. After 78 duplicates were removed, 1,219 records were screened based on review of titles and abstracts. Thereafter 63 full texts of articles were assessed for eligibility. The search to identify any missed report or citations resulted in selection of 80 articles: 61 from electronic databases search and 19 from reference lists. The reasons for 1,158 articles exclusion were: no mercury (457), without hematological effect (381), non-human (215), study type (104) and year of study (1) (Figure 1).

The Cohen's kappa statistic was considered as almost perfect $(0.98,95 \% \mathrm{CI}: 0.91 ; 1.0, \mathrm{p}<0.001)$ during screening title and abstract and substantial (0.76, 95\%CI: 0.54; 0.98, p < 0.001) during extraction process. There was no more disagreement at other steps.

All were observational studies comprising 48 case reports (42 comprising one person/52.5\%), 24 cross-sectional (12 containing control group), six case series and two cohorts. The study design was reported according to authors' description. They were published between 1950 and 2018, with an increasing pattern in the last three decades (1950: 4 studies; 1960: 5; 1970: 7; 1980: 10; 1990: 15; 2000: 19; and 2010: 20). They were done in 34 different countries (14 in the USA) on the five continents and 
Figure 1

Flowchart showing the selection of studies for the systematic review.

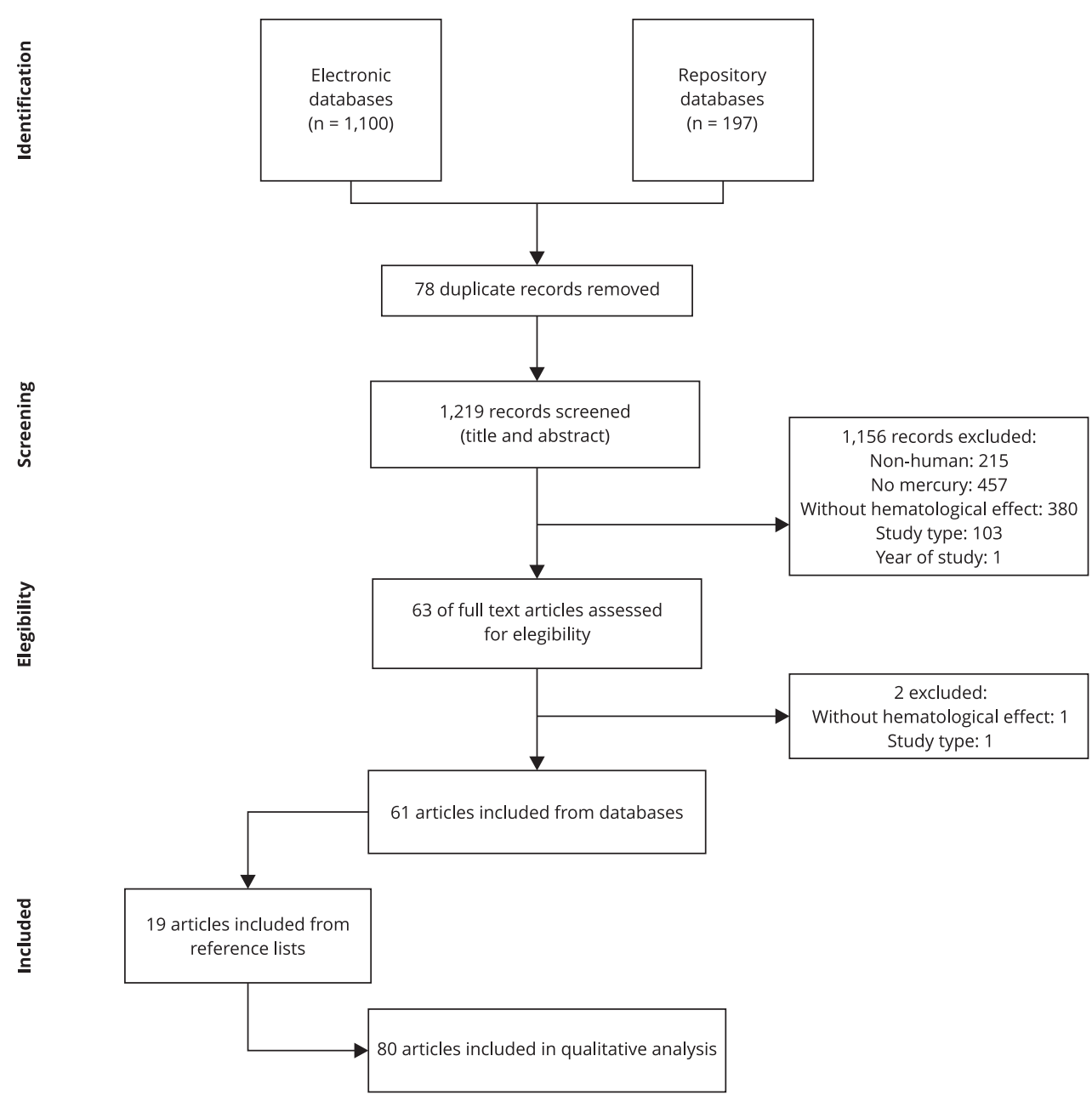

the main language was English (63). However, hematological effect was the primary outcome only in 14 studies (17.5\%).

A total of 9,284 people were evaluated: 6,601 from non-occupational (60 studies) and 2,605 from occupational (23 studies) exposure. There was no report on 78 times. Three articles had both types, so there was a split between persons, according to it.

There were differences of age and sex distribution between exposure: at non-occupational, children and teenagers were the majority $(4,982 / 75.47 \%)$ while at occupational, adults were $(1,752 / 67.26 \%)$. According to sex: women were predominant $(3,640 / 55.14 \%)$ at non-occupational and men were at occupational settings $(1,402 / 53.84 \%)$.

The non-occupational pathways of exposure were: food $(5,243)$, home near gold mining plus food (291), amalgam (454), environmental (82), medicine (54), bringing mercury home (29), suicide attempt (15), school (2), maternal exposure (2), aesthetical (1) and thermometer (1). The occupational pathways were: agriculture (1,274), gold mining (230), chlorine alkali industry (215), lamp factory (209), mix of three places: chlorine alkali industry, lamp factory and dentist's office (71), dentist's office (47) thermometer factory (2), research lab (1), fur-cleaning establishment (1) and compressor use (1). The main 
pathways of exposure among children and teenagers were: food $(4,800)$, environmental $(82)$, home near gold mining plus food (70) and bringing mercury home (14).

Three distinctive groups that are more susceptible to chemical substances, due to physiological characteristics and proportional high exposure levels, received attention on 33 studies: 23 for exposed occupational populations ( 13 cross-sectional of which seven with exposure and control groups), eight for children and teenagers (six cross-sectional and two cohorts), one for pregnant, neonates and children (cross-sectional) and one for pregnant (cross-sectional).

Chronic exposure was the main type for both, comprising 33 non-occupational (6,127 persons) and 16 occupational (2,041 persons) studies.

Hematological effects were described 2,376 times, in 69 studies comprising 1,914 cases (20.62\%): 479 children and teenagers, 476 adults, 13 elderly and 946 not classified. Non-occupational exposure was the most frequent $(1,111)$. Blood cell count was done in all cases and bone marrow biopsy in 13 times. Anemia (875), lymphocytosis (361) and lymphopenia (306) were the top three, although only anemia was the most common in both type of exposures. In fact, there was alteration of all bone marrow cellular series in mercury's presence (Table 1). In 1,567 of all cases (81.87\%), mercury's exposure biomarker was above the recommended threshold. The blood cell count was normal in 7,250 times, where 6,012 individuals were exposed to mercury (75.85\%).

Six out of seven studies reported lymphocytosis related to metallic or inorganic mercury exposure 13,22,23,24,25,26.

Fifteen studies, of which 14 are case reports $27,28,29,30,31,32,33,34,35,36,37,38,39,40$ and one is cross-sectional 41 , comprising 27 cases, reported some severe hematological effects associated to clinical condition, such as: cerebral or gastrointestinal bleeding related to thrombocytopenia (14) or aplastic anemia (2); multiple organ dysfunction syndrome and leukopenia (2) or hemolytic anemia (2) or aplastic anemia (1); renal insufficiency and thrombocytopenia (2) or leukemoid reaction (1); sepsis and aplastic anemia (2) or neutropenia (1). Mercury biomarker was measured 22 times 29,30,31,32,36,37,38,39,40,41 out of 27 and was always high according to values reported by the authors.

Death was reported 29 times, most of them at non-occupational settings (26), due to medicine use (19/26). In 19 times, the cause of death was directly related to hematological effect: 16 due to severe bleeding (thrombocytopenia [14] and aplastic anemia [2]) and three due to sepsis (neutropenia [1] and aplastic anemia[2]).

The authors of 38 studies hypothesized that mercury could be responsible for the hematological effect by direct toxicity to bone marrow (13), immunologic/hypersensitivity (9), apoptosis (5), chronic disease (3), immunologic/autoimmunity (3), inflammatory reaction (3), hemolysis (3), loss of blood (3), increased calcium content in cytoplasm (2), idiosyncrasy (2) and increased level of erythropoietin (1).

Statistical analysis concerning mercury exposure and hematological effect was reported in 17 studies as follows: 11 were mean difference (Student's $t$-test; Wilcoxon) 12,22,23,24,42,43,44,45,46,47,48; eight (two data not shown) were correlation coefficient $r$ (Pearson; Spearman) 12,22,23,24,43,44,49,50; three were regression coefficient $\beta$ (linear models) 51,52,53; two (one data not shown) were prevalence 49,54 and one was odds ratio (data not shown) 48 . The results reported for mean difference were: four studies evaluated anemia and two did not find difference $(p=0.05$ and $p=0.183) 42,48$ and other two found it $(p=0.016$ and $p<0.05) 43,47$; one study found difference for leukopenia and neutropenia $(p<0.05) 46$; five studies analyzed lymphocytes and three did not find difference ( $p$ not informed) $22,23,24$ and one did find $(\mathrm{p}<0.05) 12$; one study evaluated polycythemia and did find difference $(\mathrm{p}<0.05) 45$. For correlation coefficient, two studies evaluated anemia and found moderate negative correlation $(r=-0.4208, \mathrm{p}=0.003) 44$ and no correlation $(r=0.04$, p not informed) 49 ; six studies analyzed lymphocytes and three found no correlation $(r=-0.077$, p not informed; $r$ not informed; $r=-0.11,0.10$, p not informed) $12,23,44$ and other three found a weak to moderate positive correlation $(r=0.3405$, $\mathrm{p}<0.05 ; r=0.184, \mathrm{p}<0.05 ; r=0.121, \mathrm{p}=0.049) 22,24,50$. For regression coefficient, two studies found inverse association for lymphopenia ( $\beta=-1.26$ [95\%CI: $-2.61 ; 0.08] ; \beta=23 \%$ [95\%CI: $-43 ;-4]$ ) 51,52 and one for anemia $(\beta=-0.14, p=0.04) 53$. One found positive association for neutrophilia and basophilia $(\beta=1.38,95 \%$ CI: $0.11 ; 2.65$ and $\beta=0.04,95 \% \mathrm{CI}$ : $-0.03 ; 0.11) 51$. For prevalence, one study reported $22 \%$ (95\%CI: $18.0 ; 25.9)$ for anemia 54.

The characteristics of these studies are summarized in Tables 2 and 3. 
Table 1

Hematological effects found in the studies according to exposure.

\begin{tabular}{|c|c|c|}
\hline Hematological effect & Exposure & Cases \\
\hline \multirow[t]{2}{*}{ Anemia } & Non-occupational & 547 \\
\hline & Occupational & 328 \\
\hline \multirow[t]{2}{*}{ Polycythemia } & Non-occupational & 0 \\
\hline & Occupational & 48 \\
\hline \multirow[t]{2}{*}{ Leukopenia } & Non-occupational & 84 \\
\hline & Occupational & 22 \\
\hline \multirow[t]{2}{*}{ Leukocytosis } & Non-occupational & 23 \\
\hline & Occupational & 3 \\
\hline \multirow[t]{2}{*}{ Basopenia } & Non-occupational & 80 \\
\hline & Occupational & 0 \\
\hline \multirow[t]{2}{*}{ Basophilia } & Non-occupational & 191 \\
\hline & Occupational & 0 \\
\hline \multirow[t]{2}{*}{ Eosinophilia } & Non-occupational & 30 \\
\hline & Occupational & 7 \\
\hline \multirow[t]{2}{*}{ Neutropenia } & Non-occupational & 26 \\
\hline & Occupational & 0 \\
\hline \multirow[t]{2}{*}{ Neutrophilia } & Non-occupational & 199 \\
\hline & Occupational & 5 \\
\hline \multirow[t]{2}{*}{ Lymphopenia } & Non-occupational & 273 \\
\hline & Occupational & 33 \\
\hline \multirow[t]{2}{*}{ Lymphocytosis } & Non-occupational & 102 \\
\hline & Occupational & 259 \\
\hline \multirow[t]{2}{*}{ Monocytopenia } & Non-occupational & 56 \\
\hline & Occupational & 0 \\
\hline \multirow[t]{2}{*}{ Monocytosis } & Non-occupational & 24 \\
\hline & Occupational & 0 \\
\hline \multirow[t]{2}{*}{ Thrombocytopenia } & Non-occupational & 19 \\
\hline & Occupational & 2 \\
\hline \multirow[t]{2}{*}{ Thrombocytosis } & Non-occupational & 2 \\
\hline & Occupational & 0 \\
\hline \multirow[t]{2}{*}{ Pancytopenia } & Non-occupational & 5 \\
\hline & Occupational & 1 \\
\hline \multirow[t]{2}{*}{ Other (alteration of hematocrit and leukocytes) } & Non-occupational & 0 \\
\hline & Occupational & 7 \\
\hline Total & & 2,376 \\
\hline
\end{tabular}

The quality assessment for these studies was considered weak (3) according to EPHPP for 75 of them. Almost all component ratings were considered as weak (selection bias, study design, confounders and blinding) or not applicable (withdrawals and dropouts). There was no discrepancy between the two reviewers concerning component ratings.

\section{Discussion}

We identified 80 out of 1,219 studies of mercury exposure and hematological outcomes, including environmental studies of children, teenagers, adults and elderly, as well as occupational ones. However, there were only 14 studies that aimed at hematological effect as the primary outcome. All were observational comprising a total of 9,284 studied people, although 42 were case reports of just one person. 
Table 2

Mercury's non-occupational exposure studies.

\begin{tabular}{|c|c|c|c|c|c|c|c|}
\hline Study (year) & Country & $\begin{array}{c}\text { Study characteristics } \\
\text { [design/number/exposure] }\end{array}$ & $\begin{array}{l}\text { Population } \\
\text { [age/sex] }\end{array}$ & $\begin{array}{l}\text { Mercury } \\
\text { level }\end{array}$ & Hemtologic effect & $\begin{array}{l}\text { Statistical } \\
\text { analysis }\end{array}$ & $\begin{array}{l}\text { EPHPP } \\
\text { tool }\end{array}$ \\
\hline $\begin{array}{l}\text { Bender et al. } 27 \\
\text { (1950) }\end{array}$ & USA & $\begin{array}{l}\text { Case report; } \mathrm{n}=1 \text {; non- } \\
\text { occupational (medicine)/ } \\
\text { chronic }\end{array}$ & $\begin{array}{c}\text { Female } \\
(61 \mathrm{y})\end{array}$ & $\mathrm{NI}$ & $\begin{array}{c}\text { Anemia }=1, \\
\text { leukopenia = 1, } \\
\text { neutropenia = } 1\end{array}$ & NA & 3 \\
\hline $\begin{array}{l}\text { Butt \& } \\
\text { Simonsen } 41 \\
(1950)\end{array}$ & USA & $\begin{array}{c}\text { Cross-sectional; } \mathrm{n}=134, \\
\text { no control (occupational = } \\
1, \text { non-occupational = 55, } \\
\mathrm{NI}=78 \text { ); non-occupational } \\
\text { (medicine + environment + } \\
\text { maternal exposure) }\end{array}$ & $\begin{array}{c}\text { Female }= \\
22, \text { male }= \\
42, \mathrm{NI}=69 \\
(<1-81 \mathrm{y})\end{array}$ & High & $\begin{array}{l}\text { Thrombocytopenia } \\
=12\end{array}$ & ND & 3 \\
\hline $\begin{array}{l}\text { Doolan et al. } 73 \\
\text { (1953) }\end{array}$ & USA & $\begin{array}{c}\text { Case report; } \mathrm{n}=1 \text {; non- } \\
\text { occupational (suicide)/chronic }\end{array}$ & $\begin{array}{l}\text { Female } \\
(28 y)\end{array}$ & $\mathrm{NI}$ & Anemia $=1$ & NA & 3 \\
\hline $\begin{array}{l}\text { Portwich \& } \\
\text { Maron } 28 \text { (1959) }\end{array}$ & Germany & $\begin{array}{l}\text { Case report; } \mathrm{n}=1 \text {; non- } \\
\text { occupational (medicine)/ } \\
\text { chronic }\end{array}$ & $\begin{array}{c}\text { Female } \\
(64 y)\end{array}$ & $\mathrm{NI}$ & $\begin{array}{l}\text { Thrombocytopenia } \\
=1\end{array}$ & NA & 3 \\
\hline $\begin{array}{l}\text { Larsen et al. } 82 \\
\text { (1963) }\end{array}$ & USA & $\begin{array}{c}\text { Case report; } \mathrm{n}=2 ; \text { non- } \\
\text { occupational (medicine)/acute }\end{array}$ & $\begin{array}{c}\text { Female }= \\
1, \text { male }=1 \\
(55 y, 69 y)\end{array}$ & $\mathrm{NI}$ & $\begin{array}{l}\text { Leukocytosis }=1 \\
\quad \text { normal }=1\end{array}$ & NA & 3 \\
\hline Ross 83 (1964) & USA & $\begin{array}{l}\text { Case report; } \mathrm{n}=1 \text {; non- } \\
\text { occupational (medicine)/ } \\
\text { chronic }\end{array}$ & Male (4y) & High & Leukocytosis $=1$ & NA & 3 \\
\hline Wilson 29 (1966) & UK & $\begin{array}{l}\text { Case report; } \mathrm{n}=1 \text {; non- } \\
\text { occupational (medicine)/ } \\
\text { chronic }\end{array}$ & Male (77y) & High & $\begin{array}{c}\text { Anemia = 1, } \\
\text { leukopenia = 1, } \\
\text { thrombocytopenia } \\
=1\end{array}$ & NA & 3 \\
\hline $\begin{array}{l}\text { Johnson et al. } 30 \\
\text { (1978) }\end{array}$ & USA & $\begin{array}{l}\text { Case report; } \mathrm{n}=1 \text {; non- } \\
\text { occupational (medicine)/ } \\
\text { chronic }\end{array}$ & Female (3y) & High & $\begin{array}{c}\text { Anemia }=1 \\
\text { leukopenia }=1 \\
\text { neutropenia }=1\end{array}$ & NA & 3 \\
\hline $\begin{array}{l}\text { Hannigan } 84 \\
\text { (1978) }\end{array}$ & UK & $\begin{array}{c}\text { Case report; } \mathrm{n}=1 \text {; non- } \\
\text { occupational (suicide)/chronic }\end{array}$ & Male (34y) & High & Normal = 1 & NA & 3 \\
\hline $\begin{array}{l}\text { Murphy et al. } 31 \\
\text { (1979) }\end{array}$ & UK & $\begin{array}{c}\text { Case report; } \mathrm{n}=1 \text {; non- } \\
\text { occupational (medicine)/acute }\end{array}$ & Male (35y) & High & $\begin{array}{c}\text { Anemia = 1, } \\
\text { leukocytosis = 1, } \\
\text { neutrophilia = 1, } \\
\text { thrombocytopenia } \\
=1\end{array}$ & NA & 3 \\
\hline $\begin{array}{l}\text { Slee et al. } 32 \\
\text { (1979) }\end{array}$ & Netherlands & $\begin{array}{c}\text { Case report; } \mathrm{n}=1 ; \text { non- } \\
\text { occupational (medicine)/acute }\end{array}$ & $\begin{array}{l}\text { Female } \\
(59 y)\end{array}$ & High & $\begin{array}{c}\text { Anemia = 1, } \\
\text { leukopenia = 1, } \\
\text { thrombocytopenia } \\
=1\end{array}$ & NA & 3 \\
\hline $\begin{array}{l}\text { Wright et al. } 85 \\
\text { (1980) }\end{array}$ & UK & $\begin{array}{c}\text { Case report; } \mathrm{n}=1 \text {; non- } \\
\text { occupational (suicide)/acute }\end{array}$ & Male (17y) & $\mathrm{NI}$ & Normal = 1 & NA & 3 \\
\hline $\begin{array}{l}\text { Lien et al. } 86 \\
\text { (1983) }\end{array}$ & Canada & $\begin{array}{c}\text { Case series; } \mathrm{n}=7 \\
\text { (occupational = 1, non- } \\
\text { occupational = 6); non- } \\
\text { occupational (home)/acute }\end{array}$ & $\begin{array}{c}\text { Female }= \\
1, \text { male }= \\
2, \mathrm{NI}=3(< \\
2-28 \mathrm{y})\end{array}$ & High = 6 & Leukocytosis $=6$ & NA & 3 \\
\hline $\begin{array}{l}\text { McNeil et al. } 87 \\
(1984)\end{array}$ & UK & $\begin{array}{c}\text { Case series; } \mathrm{n}=4 \text {; non- } \\
\text { occupational (home)/chronic }\end{array}$ & $\begin{array}{l}\text { Female }= \\
2, \text { male }=2 \\
(10-41 \mathrm{y})\end{array}$ & High & Normal $=4$ & NA & 3 \\
\hline $\begin{array}{l}\text { Foulds et al. } 88 \\
\text { (1987) }\end{array}$ & USA & $\begin{array}{c}\text { Case report; } \mathrm{n}=1 \text {; non- } \\
\text { occupational (home)/chronic }\end{array}$ & $\begin{array}{c}\text { Female }(< \\
\text { 3y) }\end{array}$ & High & Normal = 1 & NA & 3 \\
\hline
\end{tabular}

(continues) 
Table 2 (continued)

\begin{tabular}{|c|c|c|c|c|c|c|c|}
\hline Study (year) & Country & $\begin{array}{c}\text { Study characteristics } \\
\text { [design/number/exposure] }\end{array}$ & $\begin{array}{l}\text { Population } \\
\text { [age/sex] }\end{array}$ & $\begin{array}{l}\text { Mercury } \\
\text { level }\end{array}$ & Hemtologic effect & $\begin{array}{l}\text { Statistical } \\
\text { analysis }\end{array}$ & $\begin{array}{l}\text { EPHPP } \\
\text { tool }\end{array}$ \\
\hline $\begin{array}{l}\text { Lauwerys et al. } \\
25 \text { (1987) }\end{array}$ & Belgium & $\begin{array}{c}\text { Case report; } \mathrm{n}=1 \text {; non- } \\
\text { occupational (aesthetic/ } \\
\text { maternal exposure)/chronic }\end{array}$ & Male (<1y) & High & $\begin{array}{c}\text { Anemia = 1, } \\
\text { leukocytosis = 1, } \\
\text { lymphocytosis = } 1\end{array}$ & NA & 3 \\
\hline $\begin{array}{l}\text { Oliveira et al. } 89 \\
\text { (1987) }\end{array}$ & UK & $\begin{array}{l}\text { Case report; } \mathrm{n}=1 \text {; non- } \\
\text { occupational (aesthetic)/ } \\
\text { chronic }\end{array}$ & $\begin{array}{l}\text { Female } \\
(46 y)\end{array}$ & High & Anemia $=1$ & NA & 3 \\
\hline $\begin{array}{l}\text { Tunnessen et } \\
\text { al. } 78 \text { (1987) }\end{array}$ & USA & $\begin{array}{c}\text { Case report; } \mathrm{n}=1 ; \text { non- } \\
\text { occupational (school)/chronic }\end{array}$ & Male $(<2 \mathrm{y})$ & High & $\begin{array}{l}\text { Leukocytosis = 1, } \\
\text { Eosinophilia = 1, } \\
\text { thrombocytosis = } 1\end{array}$ & NA & 3 \\
\hline $\begin{array}{l}\text { Murray \& } \\
\text { Hedgepeth } 90 \\
\text { (1988) }\end{array}$ & USA & $\begin{array}{c}\text { Case report; } \mathrm{n}=1 \text {; non- } \\
\text { occupational (suicide)/chronic }\end{array}$ & Male (25y) & High & $\begin{array}{c}\text { Anemia }=1 \\
\text { neutrophilia }=1\end{array}$ & NA & 3 \\
\hline $\begin{array}{l}\text { Oluwole et al. } 42 \\
\text { (1989) }\end{array}$ & Nigeria & $\begin{array}{l}\text { Cross-sectional; } \mathrm{n}=21 \text { (no } \\
\text { control); non-occupational } \\
\text { (environment)/chronic }\end{array}$ & $\begin{array}{c}\text { Male }=21 \\
(3-12 y)\end{array}$ & $\begin{array}{l}\text { High }=1 \\
\text { normal } \\
=20\end{array}$ & $\begin{array}{c}\text { Anemia }=10 \\
\text { normal }=11\end{array}$ & $\begin{array}{c}\text { t-test, } \\
\text { no mean } \\
\text { difference, } \\
p<0.05\end{array}$ & 3 \\
\hline $\begin{array}{l}\text { Siblerud } 43 \\
(1990)\end{array}$ & USA & $\begin{array}{l}\text { Cross-sectional; } \mathrm{n}=101 \\
\text { (exposed }=50, \text { not exposed } \\
=51) ; \text { non-occupational } \\
\text { (amalgam)/chronic }\end{array}$ & $\begin{array}{l}\text { Female } \\
=60, \\
\text { male }=41 \\
\text { (exposed } x \\
=22 y, \text { not } \\
\text { exposed } x \\
=23 y \text { ) }\end{array}$ & $\mathrm{NI}$ & $\begin{aligned} \text { Anemia } & =15 \\
\text { normal } & =86\end{aligned}$ & $\begin{array}{c}\text { Mean } \\
\text { difference, } \\
p=0.016 ; \\
\text { Pearson } \\
r=-0.4208 \\
p=0.003\end{array}$ & 3 \\
\hline $\begin{array}{l}\text { Montoya- } \\
\text { Cabrera et al. } 26 \\
\text { (1991) }\end{array}$ & Mexico & $\begin{array}{c}\text { Case report; } \mathrm{n}=1 ; \text { non- } \\
\text { occupational (medicine)/acute }\end{array}$ & $\begin{array}{l}\text { Female }=1 \\
\quad(<1 \mathrm{y})\end{array}$ & High & $\begin{array}{c}\text { Anemia = 1, } \\
\text { lymphocytosis = } 1\end{array}$ & NA & 3 \\
\hline $\begin{array}{l}\text { Schwartz et al. } \\
81 \text { (1992) }\end{array}$ & USA & $\begin{array}{l}\text { Case series; } \mathrm{n}=4 \text {; non- } \\
\text { occupational (home)/acute }\end{array}$ & $\begin{array}{c}\text { Female }=1 \\
\text { male }=3(< \\
4 y \text {-adults })\end{array}$ & High & $\begin{array}{l}\text { Anemia = 1, } \\
\text { leukopenia = } 1, \\
\text { thrombocytopenia } \\
=1, \text { normal }=3\end{array}$ & NA & 3 \\
\hline $\begin{array}{l}\text { Pavithran } 33 \\
\text { (1994) }\end{array}$ & India & $\begin{array}{l}\text { Case report; } \mathrm{n}=1 \text {; non- } \\
\text { occupational (medicine)/ } \\
\text { chronic }\end{array}$ & $\begin{array}{l}\text { Female } \\
(29 y)\end{array}$ & ND & $\begin{array}{c}\text { Anemia = 1, } \\
\text { leukopenia = } 1 \\
\text { thrombocytopenia } \\
=1\end{array}$ & NA & 3 \\
\hline $\begin{array}{l}\text { Alvarado et al. } \\
34 \text { (1995) }\end{array}$ & Costa Rica & $\begin{array}{c}\text { Case report; } \mathrm{n}=2 \\
\text { (occupational = 1, non- } \\
\text { occupational = 1); non- } \\
\text { occupational (medicine)/ } \\
\text { chronic }\end{array}$ & Male (25y) & $\mathrm{NI}$ & $\begin{aligned} \text { Leukocytosis } & =1 \\
\text { neutrophilia } & =1\end{aligned}$ & NA & 3 \\
\hline $\begin{array}{l}\text { Fuortes et al. } 39 \\
\text { (1995) }\end{array}$ & USA & $\begin{array}{c}\text { Case report; } \mathrm{n}=3 \text {; non- } \\
\text { occupational (home)/chronic }\end{array}$ & $\begin{array}{c}\text { Female }= \\
1, \text { male }=2 \\
(10 y, 12 y \\
17 y)\end{array}$ & High & $\begin{array}{c}\text { Anemia = 1, } \\
\text { leukocytosis = 1, } \\
\text { eosinophilia = 2, } \\
\text { thrombocytopenia } \\
=2, \text { normal = } 1\end{array}$ & NA & 3 \\
\hline $\begin{array}{l}\text { Dell'Omo et al. } \\
91 \text { (1997) }\end{array}$ & Italy & $\begin{array}{c}\text { Case report; } \mathrm{n}=1 \text {; non- } \\
\text { occupational (suicide)/chronic }\end{array}$ & Male (34y) & High & Normal = 1 & NA & 3 \\
\hline $\begin{array}{l}\text { Dada et al. } 35 \\
\text { (1999) }\end{array}$ & South Africa & $\begin{array}{c}\text { Case report; } \mathrm{n}=1 \text {; non- } \\
\text { occupational (medicine)/acute }\end{array}$ & Male (21y) & $\mathrm{NI}$ & $\begin{array}{c}\text { Anemia = 1, } \\
\text { leukopenia =1, } \\
\text { thrombocytopenia } \\
=1\end{array}$ & NA & 3 \\
\hline $\begin{array}{l}\text { Chodorowski \& } \\
\text { Anand } 92(2000)\end{array}$ & Poland & $\begin{array}{c}\text { Case report; } n=2 \text {; non- } \\
\text { occupational (suicide)/acute }\end{array}$ & $\begin{array}{l}\text { Male }=2 \\
(19-59 y)\end{array}$ & High & Normal = 2 & NA & 3 \\
\hline
\end{tabular}

(continues) 
Table 2 (continued)

\begin{tabular}{|c|c|c|c|c|c|c|c|}
\hline Study (year) & Country & $\begin{array}{c}\text { Study characteristics } \\
\text { [design/number/exposure] }\end{array}$ & $\begin{array}{l}\text { Population } \\
\text { [age/sex] }\end{array}$ & $\begin{array}{l}\text { Mercury } \\
\text { level }\end{array}$ & Hemtologic effect & $\begin{array}{l}\text { Statistical } \\
\text { analysis }\end{array}$ & $\begin{array}{l}\text { EPHPP } \\
\text { tool }\end{array}$ \\
\hline $\begin{array}{l}\text { Tschanz \& Prins } \\
77 \text { (2000) }\end{array}$ & Switzerland & $\begin{array}{c}\text { Case report; } \mathrm{n}=1 \text {; non- } \\
\text { occupational (medicine)/acute }\end{array}$ & $\begin{array}{l}\text { Female } \\
\text { (29y) }\end{array}$ & $\mathrm{NI}$ & $\begin{aligned} \text { Leukocytosis } & =1 \\
\text { eosinophilia } & =1\end{aligned}$ & NA & 3 \\
\hline $\begin{array}{l}\text { González et al. } \\
93 \text { (2001) }\end{array}$ & Venezuela & $\begin{array}{c}\text { Case report; } \mathrm{n}=1 ; \text { non- } \\
\text { occupational (medicine)/acute }\end{array}$ & Female (3y) & Normal & $\begin{array}{c}\text { Anemia }=1, \\
\text { leukocytosis }=1, \\
\text { neutrophilia }=1, \\
\text { thrombocytosis }=1\end{array}$ & NA & 3 \\
\hline $\begin{array}{l}\text { Deschamps et } \\
\text { al. } 94 \text { (2002) }\end{array}$ & France & $\begin{array}{c}\text { Case report; } \mathrm{n}=1 \text {; non- } \\
\text { occupational (suicide)/chronic }\end{array}$ & Male (41y) & High & Normal = 1 & NA & 3 \\
\hline $\begin{array}{l}\text { Langworth et } \\
\text { al. } 95 \text { (2002) }\end{array}$ & Sweden & $\begin{array}{l}\text { Cross-sectional; } \mathrm{n}=379 \text { (no } \\
\text { control); non-occupational } \\
\text { (amalgam)/chronic }\end{array}$ & $\begin{array}{l}\text { Female }= \\
263, \text { male } \\
=116(x= \\
46 y)\end{array}$ & High & $\begin{array}{c}\text { Anemia }=6 \\
\text { normal }=373\end{array}$ & ND & 3 \\
\hline $\begin{array}{l}\text { Winkler et al. } 96 \\
(2002)\end{array}$ & Austria & $\begin{array}{c}\text { Case report; } \mathrm{n}=1 ; \text { non- } \\
\text { occupational (suicide)/acute }\end{array}$ & Male (22y) & High & Anemia $=1$ & NA & 3 \\
\hline $\begin{array}{l}\text { Kouyn et al. } 97 \\
(2004)\end{array}$ & Turkey & $\begin{array}{c}\text { Case report; } \mathrm{n}=3 \text {; non- } \\
\text { occupational (home)/chronic }\end{array}$ & $\begin{array}{c}\text { Female }= \\
1, \text { male }=2 \\
(11 y, 13 y \\
16 y)\end{array}$ & $\begin{array}{l}\text { Normal } \\
=1, \text { high } \\
=2\end{array}$ & Normal = 3 & NA & 3 \\
\hline $\begin{array}{l}\text { Glezos et al. } 98 \\
\text { (2006) }\end{array}$ & Canada & $\begin{array}{c}\text { Case report; } \mathrm{n}=1 \text {; non- } \\
\text { occupational (home)/acute }\end{array}$ & Male (43y) & High & Anemia $=1$ & NA & 3 \\
\hline $\begin{array}{l}\text { Maramba et al. } \\
76(2006)\end{array}$ & Philippines & $\begin{array}{c}\text { Cross-sectional; } n=140 \\
\text { (exposed }=100, \text { not exposde } \\
=40) ; \text { non-occupational (gold } \\
\text { minig }=\text { food)/chronic }\end{array}$ & $\begin{array}{c}\text { Female }= \\
70, \mathrm{NI}=70 \\
(35<1 \mathrm{y}, 35 \\
<2 \mathrm{y}, 70 \mathrm{x}= \\
28 \mathrm{y})\end{array}$ & $\begin{array}{l}\text { Normal, } \\
\text { high * }\end{array}$ & $\begin{array}{l}\text { Anemia }=140 \\
\text { eosinophilia }=?\end{array}$ & ND & 3 \\
\hline $\begin{array}{l}\text { Frisk et al. } 46 \\
(2007)\end{array}$ & Sweden & $\begin{array}{c}\text { Cross-sectional; } n=46 \\
\text { (exposed = 24, not exposed } \\
=22) ; \text { non-occupational } \\
\text { (amalgam)/acute }\end{array}$ & Adults $=46$ & Normal & $\begin{array}{c}\text { Leukopenia }=24, \\
\text { neutropenia }=24, \\
\text { basopenia }=24, \\
\text { lymphopenia }=24, \\
\text { eosinophilia }=24, \\
\text { monocytosis }=24, \\
\text { normal }=22\end{array}$ & $\begin{array}{l}\text { Wilcoxon's } \\
\text { sign rank, } \\
\text { mean } \\
\text { difference, } \\
\text { p }<0.05\end{array}$ & 3 \\
\hline $\begin{array}{l}\text { Matushita et al. } \\
99 \text { (2007) }\end{array}$ & Brazil & $\begin{array}{c}\text { Case report; } \mathrm{n}=1 \text {; non- } \\
\text { occupational (suicide)/chronic }\end{array}$ & Male (29y) & High & Normal = 1 & NA & 3 \\
\hline $\begin{array}{l}\text { Bamonti et al. } \\
100(2008)\end{array}$ & Italy & $\begin{array}{c}\text { Case report; } \mathrm{n}=1 ; \text { non- } \\
\text { occupational (amalgam)/acute }\end{array}$ & $\begin{array}{l}\text { Female } \\
(63 y)\end{array}$ & Normal & $\begin{array}{l}\text { Anemia }=1 \\
\text { leukocytosis }=1 \\
\text { neutrophilia }=1 \\
\text { lymphopenia }=1\end{array}$ & NA & 3 \\
\hline $\begin{array}{l}\text { Girault et al. } 101 \\
\text { (2008) }\end{array}$ & France & $\begin{array}{c}\text { Case report; } \mathrm{n}=1 \text {; non- } \\
\text { occupational (medicine)/acute }\end{array}$ & $\begin{array}{l}\text { Female } \\
(66 \mathrm{y})\end{array}$ & High & $\begin{array}{l}\text { Neutrophilia }=1 \\
\text { lymphopenia }=1\end{array}$ & NA & 3 \\
\hline $\begin{array}{l}\text { Berrouet Mejía } \\
\text { et al. } 102 \text { (2008) }\end{array}$ & Colombia & $\begin{array}{c}\text { Case report; } \mathrm{n}=1 \text {; non- } \\
\text { occupational (suicide)/chronic }\end{array}$ & $\begin{array}{l}\text { Female } \\
(16 y)\end{array}$ & High & $\begin{array}{c}\text { Anemia }=1 \\
\text { leukocytosis }=1 \\
\text { neutrophilia }=1\end{array}$ & NA & 3 \\
\hline $\begin{array}{l}\text { De Palma et al. } \\
36(2008)\end{array}$ & Italy & $\begin{array}{l}\text { Case report; } \mathrm{n}=1 \text {; non- } \\
\text { occupational (suicide)/acute }\end{array}$ & $\begin{array}{l}\text { Female } \\
\text { (30y) }\end{array}$ & High & $\begin{array}{c}\text { Anemia = 1, } \\
\text { leukocytosis = } 1\end{array}$ & NA & 3 \\
\hline $\begin{array}{l}\text { Erkek et al. } 40 \\
(2010)\end{array}$ & Turkey & $\begin{array}{l}\text { Case report; } \mathrm{n}=1 \text {; non- } \\
\text { occupational (environment)/ } \\
\text { acute }\end{array}$ & $\begin{array}{l}\text { Female } \\
(10 y)\end{array}$ & High & Anemia $=1$ & NA & 3 \\
\hline $\begin{array}{l}\text { Sarikaya et al. } \\
103(2010)\end{array}$ & Turkey & $\begin{array}{l}\text { Case report; } \mathrm{n}=1 \text {; non- } \\
\text { occupational (school)/acute }\end{array}$ & $\begin{array}{l}\text { Female } \\
(36 y)\end{array}$ & $\mathrm{NI}$ & Normal = 1 & NA & 3 \\
\hline
\end{tabular}

(continues) 
Table 2 (continued)

\begin{tabular}{|c|c|c|c|c|c|c|c|}
\hline Study (year) & Country & $\begin{array}{c}\text { Study characteristics } \\
\text { [design/number/exposure] }\end{array}$ & $\begin{array}{l}\text { Population } \\
\text { [age/sex] }\end{array}$ & $\begin{array}{c}\text { Mercury } \\
\text { level }\end{array}$ & Hemtologic effect & $\begin{array}{l}\text { Statistical } \\
\text { analysis }\end{array}$ & $\begin{array}{l}\text { EPHPP } \\
\text { tool }\end{array}$ \\
\hline $\begin{array}{l}\text { Al-Sinani et al. } \\
79 \text { (2011) }\end{array}$ & Oman & $\begin{array}{l}\text { Case report; } \mathrm{n}=1 \text {; non- } \\
\text { occupational (medicine)/ } \\
\text { chronic }\end{array}$ & $\begin{array}{l}\text { Female } \\
(12 \mathrm{y})\end{array}$ & High & $\begin{aligned} \text { Leukocytosis } & =1 \\
\text { eosinophilia } & =1\end{aligned}$ & NA & 3 \\
\hline $\begin{array}{l}\text { Plante et al. } 49 \\
\text { (2011) }\end{array}$ & Canada & $\begin{array}{l}\text { Cross-sectional; } n=466 \text { (no } \\
\text { control); non-occupational } \\
\text { (food)/chronic }\end{array}$ & $\begin{array}{c}\text { Female = } \\
466(20- \\
54 y)\end{array}$ & High & $\begin{aligned} \text { Anemia } & =200 \\
\text { normal } & =266\end{aligned}$ & $\begin{array}{c}\text { Previous } \\
\text { data not } \\
\text { shown. } \\
\text { Pearson } \\
r=0.04 \\
\text { p-value not } \\
\text { informed }\end{array}$ & 3 \\
\hline $\begin{array}{l}\text { Yildrim et al. } 37 \\
\text { (2012) }\end{array}$ & Turkey & $\begin{array}{l}\text { Case series; } \mathrm{n}=5 \text {; non- } \\
\text { occupational (home)/acute }\end{array}$ & $\begin{array}{c}\text { Female }= \\
3, \text { male }=2 \\
(20-54 \mathrm{y})\end{array}$ & High & $\begin{array}{c}\text { Anemia }=2, \\
\text { leukopenia }=2, \\
\text { thrombocytopenia } \\
=2, \text { normal }=2\end{array}$ & NA & 3 \\
\hline $\begin{array}{l}\text { Priya et al. } 11 \\
\text { (2012) }\end{array}$ & India & $\begin{array}{c}\text { Case report; } \mathrm{n}=1 ; \text { non- } \\
\text { occupational (suicide)/acute }\end{array}$ & $\begin{array}{l}\text { Female } \\
(19 y)\end{array}$ & High & $\begin{array}{c}\text { Anemia = 1, } \\
\text { leukopenia =1, } \\
\text { thrombocytopenia } \\
=1\end{array}$ & NA & 3 \\
\hline $\begin{array}{l}\text { Khoury et al. } 104 \\
\text { (2013) }\end{array}$ & Brazil & $\begin{array}{c}\text { Cross-sectional; } n=157 \\
\text { (exposed = 108, not exposed } \\
=49 \text { ); non-occupational (gold } \\
\text { mining + food)/chronic }\end{array}$ & $\begin{array}{l}\mathrm{NI}=157 \\
(13-53 \mathrm{y})\end{array}$ & $\begin{array}{l}\text { Normal = } \\
49, \text { high } \\
=108\end{array}$ & $\mathrm{NI}$ & ND & 3 \\
\hline $\begin{array}{l}\text { Kim et al. } 50 \\
\text { (2013) }\end{array}$ & Korea & $\begin{array}{l}\text { Cross-sectional; n = } 311 \text { (no } \\
\text { control); non-occupational } \\
\text { (food)/chronic }\end{array}$ & $\begin{array}{c}\text { Female = } \\
141, \text { male }= \\
170(5-12 y)\end{array}$ & High = 7 & $\begin{array}{l}\text { Lymphocytosis = } \\
100, \text { normal = } 211\end{array}$ & $\begin{array}{c}\text { Spearman } r \\
=0.121, p= \\
0.049\end{array}$ & 3 \\
\hline $\begin{array}{l}\text { Wu et al. } 72 \\
(2013)\end{array}$ & Taiwan & $\begin{array}{c}\text { Case report; } \mathrm{n}=1 \text {; non- } \\
\text { occupational (medicine)/acute }\end{array}$ & Male (51y) & Normal & Anemia $=1$ & NA & 3 \\
\hline $\begin{array}{l}\text { Beasley et al. } \\
105 \text { (2014) }\end{array}$ & $\begin{array}{l}\text { New } \\
\text { Zealand }\end{array}$ & $\begin{array}{c}\text { Case report; } \mathrm{n}=1 ; \text { non- } \\
\text { occupational (suicide)/acute }\end{array}$ & $\begin{array}{l}\text { Female } \\
(19 y)\end{array}$ & High & $\begin{aligned} \text { Leukocytosis } & =1 \\
\text { neutrophilia } & =1\end{aligned}$ & NA & 3 \\
\hline $\begin{array}{l}\text { Brázdová et al. } \\
47 \text { (2014) }\end{array}$ & $\begin{array}{l}\text { Kazakhstan, } \\
\text { Kyrgyzstan, } \\
\text { Uzbeskistan }\end{array}$ & $\begin{array}{l}\text { Cross-sectional; } \mathrm{n}=60 \text {; non- } \\
\text { occupational (environment)/ } \\
\text { chronic }\end{array}$ & $\begin{array}{l}\text { Female }= \\
27, \text { male }= \\
33(1-15 y)\end{array}$ & Normal & Anemia $=60$ & $\begin{array}{l}\text { Bonferroni's } \\
\text { test, mean } \\
\text { difference, } \\
p<0.05\end{array}$ & 3 \\
\hline $\begin{array}{l}\text { Dardamanis et } \\
\text { al. } 106(2014)\end{array}$ & Greece & $\begin{array}{c}\text { Case report; } \mathrm{n}=1 \text {; non- } \\
\text { occupational (thermometer)/ } \\
\text { acute }\end{array}$ & $\begin{array}{l}\text { Female } \\
(48 \mathrm{y})\end{array}$ & $\mathrm{NI}$ & Anemia $=1$ & NA & 3 \\
\hline $\begin{array}{l}\text { Cicek-Senturk } \\
\text { et al. } 107 \text { (2014) }\end{array}$ & Turkey & $\begin{array}{c}\text { Case report; } \mathrm{n}=3 \text {; non- } \\
\text { occupational (home)/chronic }\end{array}$ & $\begin{array}{c}\text { Female }= \\
1, \text { male }=2 \\
(14 y, 52 y \\
\text { NI })\end{array}$ & High & $\begin{array}{c}\text { Anemia = 1, } \\
\text { leukocytosis = } 3\end{array}$ & NA & 3 \\
\hline $\begin{array}{l}\text { Mathee et al. } 48 \\
\text { (2014) }\end{array}$ & South Africa & $\begin{array}{l}\text { Cross-sectional; } \mathrm{n}=307 \\
\text { (exposed }=60, \text { not exposed } \\
=247) ; \text { non-occupational } \\
\text { (geophagia)/chronic }\end{array}$ & $\begin{array}{c}\text { Female = } \\
307(18- \\
46 y)\end{array}$ & Normal & $\begin{array}{l}\text { Anemia }=52 \\
\text { normal }=255\end{array}$ & $\begin{array}{c}\text { No } \\
\text { association } \\
\text { (OR not } \\
\text { informed), } \\
\text { no mean } \\
\text { difference, } \\
p=0.183\end{array}$ & 2 \\
\hline $\begin{array}{l}\text { Kim et al. } 51 \\
(2015)\end{array}$ & Korea & $\begin{array}{c}\text { Cohort; } \mathrm{n}=4,350 \text { (no control); } \\
\text { non-occupational (food)/ } \\
\text { chronic }\end{array}$ & $\begin{array}{c}\text { Female }= \\
2,175, \text { male } \\
=2,175(7 y)\end{array}$ & High & $\begin{array}{c}\text { Basophilia = 191, } \\
\text { neutrophilia = } \\
\text { 191, lymphopenia } \\
=191, \text { normal = } \\
4,159\end{array}$ & $\begin{array}{c}\text { Linear } \\
\text { mixed } \\
\text { model, } \beta= \\
1.38(95 \% \mathrm{Cl} \text { : } \\
0.11 ; 2.65)\end{array}$ & 2 \\
\hline
\end{tabular}

(continues) 
Table 2 (continued)

\begin{tabular}{|c|c|c|c|c|c|c|c|}
\hline Study (year) & Country & $\begin{array}{c}\text { Study characteristics } \\
\text { [design/number/exposure] }\end{array}$ & $\begin{array}{l}\text { Population } \\
\text { [age/sex] }\end{array}$ & $\begin{array}{l}\text { Mercury } \\
\text { level }\end{array}$ & Hemtologic effect & $\begin{array}{l}\text { Statistical } \\
\text { analysis }\end{array}$ & $\begin{array}{l}\text { EPHPP } \\
\text { tool }\end{array}$ \\
\hline $\begin{array}{l}\text { Oulhote et al. } 52 \\
\text { (2017) }\end{array}$ & Denmark & $\begin{array}{c}\text { Cohort; n = } 56 \text { (no control); } \\
\text { non-occupational (food)/ } \\
\text { chronic }\end{array}$ & $\begin{array}{c}\text { Female = } \\
33, \text { male = } \\
22, \mathrm{NI}=1 \\
(7-12 \mathrm{y})\end{array}$ & $\begin{array}{l}\text { Normal, } \\
\text { high * }\end{array}$ & $\begin{array}{c}\text { Leukopenia = 56, } \\
\text { basopenia = 56, } \\
\text { lymphopenia = 56, } \\
\text { monocytopenia } \\
=56\end{array}$ & $\begin{array}{c}\text { Structural } \\
\text { equation, } \\
\beta=-0.23 \\
(95 \% \mathrm{Cl} \text { : } \\
-043 ;-0.04) \text {, } \\
p=0.02\end{array}$ & 2 \\
\hline $\begin{array}{l}\text { Weinhouse et } \\
\text { al. } 53 \text { (2017) }\end{array}$ & Peru & $\begin{array}{l}\text { Cross-sectional; n = } 83 \text { (no } \\
\text { control); non-occupational } \\
\text { (gold mining + food)/chronic }\end{array}$ & $\begin{array}{c}\text { Female }= \\
44, \text { male }= \\
38, \mathrm{NI}=1(< \\
12 \mathrm{y})\end{array}$ & $\begin{array}{l}\text { Normal = } \\
\text { 35, high } \\
=48\end{array}$ & $\begin{aligned} \text { Anemia } & =41 \\
\text { normal } & =42\end{aligned}$ & $\begin{array}{l}\text { Multivariate } \\
\text { linear, } \\
\text { regression } \\
\text { model, } \beta= \\
-0.14 \mathrm{~g} / \mathrm{dL} \text {, } \\
\mathrm{p}=0.04\end{array}$ & 2 \\
\hline
\end{tabular}

95\%Cl: 95\% confidence interval; EPHPP: Effective Public Health Practice Project; NA: not applicable; ND: not done; NI: not informed.

* From normal to high levels of mercury (range).

It is important to emphasize the growing articles publication involving human exposure to chemical substances over the past decades. This is a consequence of the efforts made by many countries, through their agencies and institutions, in order to improve health by reducing environmental exposure to toxic substances 55,56. Mercury is no exception, as in this review, we reported 26 studies published between the 1950s and the 1980s and 54 studies in the last three decades 57.

Distribution by age and sex presented the results expected in the literature, where children/ teenagers and women were more commonly exposed non-occupationally, while adults and men were occupationally exposed. According to the report on human exposure to environmental chemicals (National Health and Nutrition Examination Survey IV - NHANES), women are the most exposed on non-occupational setting 8 . The main exposure pathway for children/teenagers was food consumption, mainly fish and shellfish, although rice may be another methylmercury source for Asians 58 . Another concern regarding this group is the fact that this silver liquid metal - found at home, at school and at others sites where it is not adequately stored - is seen by them as an amusing substance to play with, which may cause health problems. Lee et al. 59 addressed this subject by reviewing the sources of mercury exposures in children, the location and proportion of children affected and also making recommendations to prevent them.

There was a wide range of exposure pathway, from food and medicine intake, suicide attempt to industrial process and gold mining, among others. Many of them are supposed to be prohibited by 2020, according to Minamata Convention, an international treaty signed in 2013 by more than 140 countries, including Brazil, which have committed to eliminate the use of mercury in different products, such as batteries, light bulbs and health equipment ${ }^{2}$. Two of them deserve a special attention: gold mining/ASGM and fish/shellfish consumption, since they play a role in both types of exposure, occupational and non-occupational.

The first exposure pathway, gold mining/ASGM, is the main anthropogenic mercury pollutant in the world, affecting not only the miners but also the neighboring population, mainly in Southeast/East Asia, Sub-Saharan and South America 2. It is impressive that only seven research articles (four occupational and three non-occupational) have addressed hematological effects among people working or living nearby the gold mining sector, as more than 10 million ASGM miners, most of them informally or even illegally 5 , are exposed to mercury through both direct inhalation of mercury vapor and consumption of material taken from contaminated areas (e.g. fish). One example addressing this topic was the research, a purposive field sampling, conducted in Indonesia by Ekawanti \& Krisnayanti 60 among non-miners (29) and miners (71), who showed lower levels of hemoglobin and hematocrit. In non-occupational situations, houses near gold mining put the surrounding population at risk due 
Table 3

Mercury's occupational exposure studies.

\begin{tabular}{|c|c|c|c|c|c|c|c|}
\hline Study (year) & Country & $\begin{array}{c}\text { Study characteristics } \\
\text { [design/number/exposure] }\end{array}$ & $\begin{array}{l}\text { Population } \\
\text { [age/sex] }\end{array}$ & $\begin{array}{l}\text { Mercury } \\
\text { level }\end{array}$ & Hemtologic effect & $\begin{array}{l}\text { Statistical } \\
\text { analysis }\end{array}$ & $\begin{array}{l}\text { EPHHP } \\
\text { tool }\end{array}$ \\
\hline $\begin{array}{l}\text { Butt \& } \\
\text { Simonsen } 41 \\
(1950)\end{array}$ & USA & $\begin{array}{c}\text { Cross-sectional; } \mathrm{n}=134, \\
\text { no control (occupational = } \\
\text { 1, non-occupational = 55, } \\
\mathrm{NI}=78 \text { ); occupational (fur } \\
\text { cleaning plant)/chronic }\end{array}$ & Female (49y) & High & $\begin{array}{l}\text { Thrombocytopenia } \\
=1\end{array}$ & ND & 3 \\
\hline $\begin{array}{l}\text { Devlin \& } \\
\text { Sudlow } 108 \\
\text { (1967) }\end{array}$ & UK & $\begin{array}{c}\text { Case report; } \mathrm{n}=1 ; \\
\text { occupational (laboratory)/ } \\
\text { chronic }\end{array}$ & Male (32y) & High & $\begin{array}{l}\text { Leukopenia = 1, } \\
\text { thrombocytopenia } \\
=1\end{array}$ & NA & 3 \\
\hline $\begin{array}{l}\text { Takamatsu et } \\
\text { al. } 109 \text { (1969) }\end{array}$ & Japan & $\begin{array}{c}\text { Case series; } \mathrm{n}=5 ; \\
\text { occupational (agriculture)/ } \\
\text { chronic }\end{array}$ & $\begin{array}{c}\text { Male }=5 \\
\text { (adults }=5)\end{array}$ & High $=5$ & Leukopenia $=5$ & NA & 3 \\
\hline $\begin{array}{l}\text { Ryrie et al. } 38 \\
\text { (1970) }\end{array}$ & UK & $\begin{array}{c}\text { Case report; } \mathrm{n}=1 ; \\
\text { occupational (thermometer } \\
\text { fabric)/acute }\end{array}$ & Male (59y) & High & $\begin{array}{c}\text { Anemia }=1 \\
\text { leukopenia }=1 \\
\text { thrombocytopenia } \\
=1\end{array}$ & NA & 3 \\
\hline $\begin{array}{l}\text { Gys \& Fadeev } \\
80 \text { (1971) }\end{array}$ & USSR & $\begin{array}{l}\text { Cross-sectional; } \mathrm{n}=103 \\
\text { (no control); occupational } \\
\text { (agriculture)/chronic }\end{array}$ & $\begin{array}{c}\text { NI (adults = } \\
103)\end{array}$ & ND & Leukopenia = ? & ND & 3 \\
\hline $\begin{array}{l}\text { Nizov \& } \\
\text { Shestakov } 110 \\
(1971)\end{array}$ & USSR & $\begin{array}{c}\text { Case series; } \mathrm{n}=10 ; \\
\text { occupational (agriculture)/ } \\
\text { acute }\end{array}$ & $\begin{array}{l}\text { NI (adults } \\
\quad=10)\end{array}$ & ND & $\begin{array}{c}\text { Anemia }=10 \\
\text { leukopenia }=10\end{array}$ & NA & 3 \\
\hline $\begin{array}{l}\text { Jung \& } \\
\text { Aaronson } 111 \\
\text { (1980) }\end{array}$ & USA & $\begin{array}{c}\text { Case report; } \mathrm{n}=1 ; \\
\text { occupational (gold mining)/ } \\
\text { acute }\end{array}$ & Male (53y) & High & $\begin{array}{l}\text { Polycythemia }=1 \\
\text { leukocytosis }=1 \\
\text { neutrophilia }=1\end{array}$ & NA & 3 \\
\hline $\begin{array}{l}\text { Lien et al. } 86 \\
\text { (1983) }\end{array}$ & Canada & $\begin{array}{c}\text { Case series; } \mathrm{n}=7 \\
\text { (occupational }=1, \\
\text { non-occupational }=6 \text { ); } \\
\text { occupational (gold mining)/ } \\
\text { acute }\end{array}$ & $\begin{array}{c}\text { Male = } 1 \\
(28 y)\end{array}$ & High $=1$ & Leukocytosis $=1$ & NA & 3 \\
\hline $\begin{array}{l}\text { Kanamaru et } \\
\text { al. } 75 \text { (1984) }\end{array}$ & Japan & $\begin{array}{c}\text { Cross-sectional; } \mathrm{n}=1,164 \\
\text { (no control); occupational } \\
\text { (agriculture)/chronic }\end{array}$ & $\begin{array}{c}\text { Female }= \\
397, \text { male }= \\
767 \text { (adults }= \\
1,164)\end{array}$ & $\begin{array}{c}\text { Female } x= \\
2.97, \text { male } \\
x=5.35\end{array}$ & $\begin{aligned} \text { Anemia } & =197, \text { normal } \\
& =967\end{aligned}$ & ND & 3 \\
\hline $\begin{array}{l}\text { Langworth et } \\
\text { al. } 44 \text { (1993) }\end{array}$ & Sweden & $\begin{array}{c}\text { Cross-sectional; } \mathrm{n}=110 \\
\text { (exposed }=71, \text { not exposed }= \\
\text { 39); occupational (chlor-alkali } \\
\text { fabric/lamp facgtory/dentist } \\
\text { office)/chronic }\end{array}$ & $\begin{array}{l}\text { Female } 75 \\
\text { male }=35 \\
\text { (exposed } \mathrm{x} \\
=49 \mathrm{y}, \mathrm{not} \\
\text { exposed } \mathrm{x}= \\
40 \mathrm{y})\end{array}$ & $\begin{array}{l}\text { Exposed } x \\
=4.3, \text { not } \\
\text { exposed } x \\
=3.9\end{array}$ & Normal $=110$ & $\begin{array}{c}\text { t-test, } \\
\text { no mean } \\
\text { difference, } \\
\text { Pearson } \\
\text { r not } \\
\text { informed, } \\
\text { no } \\
\text { correlation }\end{array}$ & 3 \\
\hline $\begin{array}{l}\text { Torresani et } \\
\text { al. } 112 \text { (1993) }\end{array}$ & Italy & $\begin{array}{c}\text { Case report; } \mathrm{n}=1 ; \\
\text { occupational (agriculture)/ } \\
\text { chronic }\end{array}$ & Female (54y) & ND & Eosinophilia $=1$ & NA & 3 \\
\hline $\begin{array}{l}\text { Zavariz \& } \\
\text { Glina } 13 \text { (1993) }\end{array}$ & Brazil & $\begin{array}{l}\text { Cross-sectional; n = } 91 \text { (no } \\
\text { control); occupational (lamp } \\
\text { fabric)/chronic }\end{array}$ & $\begin{array}{c}\text { Female }=8 \\
\text { male }=83 \\
(20-65 y)\end{array}$ & $\begin{array}{l}\text { High }=54 \\
\text { normal }= \\
32, \mathrm{NI}=42\end{array}$ & $\begin{array}{c}\text { Anemia = 1, } \\
\text { leukopenia = 6, } \\
\text { lymphocytosis = 6, } \\
\text { eosinophilia = 5, } \\
\text { neutrophilia = 4, } \\
\text { normal = } 69\end{array}$ & ND & 3 \\
\hline
\end{tabular}

(continues) 
Table 3 (continued)

\begin{tabular}{|c|c|c|c|c|c|c|c|}
\hline Study (year) & Country & $\begin{array}{c}\text { Study characteristics } \\
\text { [design/number/exposure] }\end{array}$ & $\begin{array}{c}\text { Population } \\
\text { [age/sex] }\end{array}$ & $\begin{array}{l}\text { Mercury } \\
\text { level }\end{array}$ & Hemtologic effect & $\begin{array}{c}\text { Statistical } \\
\text { analysis }\end{array}$ & $\begin{array}{c}\text { EPHHP } \\
\text { tool }\end{array}$ \\
\hline $\begin{array}{l}\text { Moszczyński } \\
\text { \& Slowiński } 22 \\
\text { (1994) }\end{array}$ & Poland & $\begin{array}{c}\text { Cross-sectional; } \mathrm{n}=91 \\
\text { (exposed }=55, \text { not exposed = } \\
\text { 36); occupational (chlor-alkali } \\
\text { fabric)/chronic }\end{array}$ & $\begin{array}{l}\text { Male = } 91 \\
(28-55 y)\end{array}$ & $\begin{array}{l}\text { High }=55 \\
\text { normal } \\
=36\end{array}$ & $\begin{array}{c}\text { Lymphocytosis }=55 \\
\text { normal }=36\end{array}$ & $\begin{array}{c}\text { t-test, } \\
\text { no mean } \\
\text { difference, } \\
\text { Pearson } \\
r=0.3405, \\
p<0.05\end{array}$ & 3 \\
\hline $\begin{array}{l}\text { Alvarado et al. } \\
34 \text { (1995) }\end{array}$ & Costa Rica & $\begin{array}{c}\text { Case report; } \mathrm{n}=2 \\
\text { (occupational = } 1 \\
\text { non-occupational = 1); } \\
\text { occupational (compressor)/ } \\
\text { chronic }\end{array}$ & Male (30y) & High & Normal $=1$ & NA & 3 \\
\hline $\begin{array}{l}\text { Moszczyński } \\
\text { et al. } 23 \text { (1995) }\end{array}$ & Poland & $\begin{array}{c}\text { Cross-sectional; } \mathrm{n}=117 \\
\text { (exposed }=81, \text { not exposed = } \\
\text { 36); occupational (chlor-alkali } \\
\text { fabric)/chronic }\end{array}$ & $\begin{array}{c}\text { Male }=117 \\
(21-60 y)\end{array}$ & $\begin{array}{l}\text { Exposed } \mathrm{x} \\
\quad=54\end{array}$ & $\begin{array}{c}\text { Lymphocytosis }=81 \\
\text { normal }=36\end{array}$ & $\begin{array}{c}\text { t-test, } \\
\text { no mean } \\
\text { difference, } \\
\text { Pearson } \\
r=-0.11, \\
0.10, \text { no } \\
\text { correlation }\end{array}$ & 3 \\
\hline $\begin{array}{l}\text { Queiroz \& } \\
\text { Dantas } 12 \\
(1997)\end{array}$ & Brazil & $\begin{array}{c}\text { Cross-sectional; } n=41 \\
\text { (exposed = 33, not exposed = } \\
\text { 8); occupational (chlor-alkali } \\
\text { fabric)/chronic }\end{array}$ & NI (19-46y) & $\begin{array}{l}\text { Normal } \\
=41\end{array}$ & $\begin{array}{c}\text { Lymphopenia = 33, } \\
\text { normal }=8\end{array}$ & $\begin{array}{c}\text { Mann- } \\
\text { Whitney's } \\
U \text {, mean } \\
\text { difference, } \\
\mathrm{p}<0.05 \text {, } \\
\text { Pearson } r \\
=-0.077 \text {, } \\
\text { no } \\
\text { correlation }\end{array}$ & 3 \\
\hline $\begin{array}{l}\text { Melo et al. } 113 \\
(2000)\end{array}$ & Venezuela & $\begin{array}{l}\text { Cross-sectional; } \mathrm{n}=47 \\
\text { (no control); occupational } \\
\text { (dentist office)/chronic }\end{array}$ & $\mathrm{NI}$ & $\mathrm{NI}$ & $\begin{array}{c}\text { Hemoglobin } \\
\text { alteration }=4, \\
\text { leukocyte alteration }= \\
3, \text { normal }=40\end{array}$ & ND & 3 \\
\hline $\begin{array}{l}\text { Zabiński et al. } \\
45 \text { (2000) }\end{array}$ & Poland & $\begin{array}{c}\text { Cross-sectional; } \mathrm{n}=81 \\
\text { (exposed }=46, \text { not exposed = } \\
\text { 35); occupational (chlor-alkali } \\
\text { fabric)/chronic }\end{array}$ & $\mathrm{NI}(20-56 y)$ & $\begin{array}{c}x=77.44 \\
\pm 48.15 \\
\text { (exposed/ } \\
\text { not } \\
\text { exposed) }\end{array}$ & $\begin{array}{c}\text { Polycythemia }=46 \\
\text { normal }=35\end{array}$ & $\begin{array}{c}\text { t-test, } \\
\text { mean } \\
\text { difference, } \\
47.89 \% \text { vs. } \\
46.1 \% \\
\mathrm{p}<0.05\end{array}$ & 3 \\
\hline $\begin{array}{l}\text { Soleo et al. } 24 \\
(2002)\end{array}$ & Italy & $\begin{array}{c}\text { Cross-sectional; } \mathrm{n}=289 \\
\text { (exposed = 117, not exposed } \\
=172 \text { ); occupational (lamp } \\
\text { fabric)/chronic }\end{array}$ & $\begin{array}{c}\mathrm{NI} \text { (adults = } \\
\text { 289) }\end{array}$ & $\begin{array}{c}\text { Normal }= \\
289\end{array}$ & $\begin{array}{c}\text { Lymphocytosis }=117 \\
\text { normal }=172\end{array}$ & $\begin{array}{c}\text { t-test, } \\
\text { no mean } \\
\text { difference, } \\
\text { Pearson } \\
r=0.184, \\
\text { positive } \\
\text { correlation }\end{array}$ & 3 \\
\hline $\begin{array}{l}\text { Campbell et } \\
\text { al. } 74 \text { (2009) }\end{array}$ & UK & $\begin{array}{l}\text { Case report; } \mathrm{n}=1 ; \\
\text { occupational (lamp fabric)/ } \\
\text { acute }\end{array}$ & Male (25y) & High & Polycythemia = 1 & NA & 3 \\
\hline
\end{tabular}

(continues) 
Table 3 (continued)

\begin{tabular}{|c|c|c|c|c|c|c|c|}
\hline Study (year) & Country & $\begin{array}{c}\text { Study characteristics } \\
\text { [design/number/exposure] }\end{array}$ & $\begin{array}{l}\text { Population } \\
\text { [age/sex] }\end{array}$ & $\begin{array}{l}\text { Mercury } \\
\text { level }\end{array}$ & Hemtologic effect & $\begin{array}{l}\text { Statistical } \\
\text { analysis }\end{array}$ & $\begin{array}{l}\text { EPHHP } \\
\text { tool }\end{array}$ \\
\hline $\begin{array}{l}\text { Rodríguez et } \\
\text { al. } 114 \text { (2002) }\end{array}$ & Spain & $\begin{array}{l}\text { Cross-sectional; } \mathrm{n}=26 \text { (no } \\
\text { control); occupational (gold } \\
\text { mining)/chronic }\end{array}$ & $\mathrm{NI}$ & ND & Anemia $=26$ & NA & 3 \\
\hline $\begin{array}{l}\text { Alhamad et al. } \\
115 \text { (2011) }\end{array}$ & USA & $\begin{array}{c}\text { Case report; } \mathrm{n}=1 \\
\text { occupational (thermometer } \\
\text { fabric)/acute }\end{array}$ & Male $=(36 y)$ & High & $\begin{aligned} \text { Leukocytosis } & =1 \\
\text { eosinophilia } & =1\end{aligned}$ & NA & 3 \\
\hline $\begin{array}{l}\text { Douine et al. } \\
54 \text { (2018) }\end{array}$ & $\begin{array}{l}\text { Guyana/ } \\
\text { Suriname }\end{array}$ & $\begin{array}{c}\text { Cross-sectional; } \mathrm{n}=421 \\
\text { (exposed }=202, \text { not exposed } \\
=219 \text { ); occupational (gold } \\
\text { mining)/chronic }\end{array}$ & $\begin{array}{c}\text { Female }= \\
124, \text { male }= \\
297(x=37 y)\end{array}$ & ND & $\begin{aligned} \text { Anemia } & =93, \text { normal } \\
& =328\end{aligned}$ & $\begin{array}{l}\text { Prevalence } \\
\qquad 22 \% \\
(95 \% \mathrm{Cl}: \\
18.0 ; 25.9)\end{array}$ & 2 \\
\hline
\end{tabular}

95\%Cl: 95\% confidence interval; EPHPP: Effective Public Health Practice Project; NA: not applicable; ND: not done; NI: not informed.

to contamination of soil (children playing outdoor), water (fish consumption) and air (amalgamation process or re-burning it at gold shops) 5. An example of the latter was a study carried out in Poconé, a town in Mato Grosso State/Brazil 61. They evaluated the levels of exposure to metallic mercury emissions by gold dealers and its health effects. It was reported higher mercury levels and referred morbidity among downtown residents.

The second route of exposure, consumption of fish and shellfish, is a major concern for regulatory agencies around the world, because although it is an important part of a healthy diet (presence of omega-3 fatty acids and low in saturated fat), it is also cited as the most significant source of methylmercury. One of the agencies is the U.S. Environmental Protective Agency (EPA), who sets a recommendation to limit or avoid certain species of fish and shellfish for general public and for specific groups of people at risk, such as: high consumers of fish (e.g. coastal dwellers, riverside communities), women of childbearing age, pregnant and breastfeeding women and young children. For example, the threshold for tuna consumption, a carnivorous fish, is one can (226-340g) per week for groups of people at risk 62 . The risk of contamination of this kind of food is usually high (especially for the species at the top of food chain), because of the bioavailability of this metal in the aquatic environment from different sources such as geothermal activities, fossil fuel burning, hazardous waste incineration, industrial processes, gold mining and so forth. The ASGM, despite its decline in Amazon Basin, continues to contribute to an increase in the mercury load, becoming a major risk for indigenous groups and riverside communities, who have fish as their main source of protein 63 . On other hand, the general urban population has a low fish ingestion as a result of its cultural and social characteristics, in such a way that they do not face significant health effects from this pathway exposure 64,65. However, the fish resources for urban centers may come from a contaminated water body, as reported by Hacon et al. 64 in a study carried out in Alta Floresta, a town in Mato Grosso State/ Brazil. The assessment of the impact of fish and shellfish contamination on the exposure of human beings and on their health through food deserves special attention, specifically, but not only, for those who are large consumers, such as indigenous groups, riverside communities (e.g. Amazon Basin), coastal (e.g. Florida/Puerto Rico) and island dwellers (e.g. Faroe Islands/Denmark). In this review, 3 articles have targeted this population and hematological effects: 1 sectional study with an indigenous group from the Peruvian Amazon near ASGM, that reported anemia among children under 12 years (83 persons) 66; 1 cohort with children from Faroe Islands (56 persons), that reported leukopenia and lymphopenia 52 , and other sectional with children from Jeju Island/South Korea (311 persons), that reported lymphocytosis 50 .

Anemia and less commonly leukopenia, eosinophilia, thrombocytopenia and pancytopenia have been reported due to mercury toxicity 67 . In this review, most exposed people $(75.85 \%)$ had a normal 
blood cell count, however, hematological effects were reported 2,376 times, mainly at non-occupational settings, comprising 1,914 cases. All bone marrow cellular series were affected and the most common, for both exposures, was the erythroid series with anemia (875). Out of five studies that addressed this subject using statistical analysis with significant p-value, there was mean difference in two 43,47, none in one study 42; negative correlation 42 was reported in one and inverse association 53 in another. On the other hand, polycythemia was also reported in the mercury exposure group and a mean difference was found 45 . Other two hematological effects were also reported: lymphocytosis and lymphopenia. For the first outcome, there were three studies that reported a weak to moderate positive correlation 22,24,50 between lymphocytes and mercury. For the latter, one reported mean difference 12 and other two described inverse association between lymphocytes and mercury exposure 51,52. One of these studies also described an association between mercury exposure and an increased neutrophils and basophils percentage 51. These results confirm there are relation or association between mercury exposure and hematological effects, especially for anemia, lymphopenia, lymphocytosis, neutrophilia and basophilia. However, none of these studies could determine a causal relationship, as they were not designed for this purpose.

Recently, researches shed some light on the role of heavy metal exposure at anemia, which is estimated by the World Health Organization (WHO) in 1.62 billion cases (95\%CI: 1.0; 1.74 billion) 47,48,49. More than half of the cases are caused by iron deficit (51\%) and current data point to relation between heavy metals, such as lead and mercury, and iron metabolism (positive correlation for mercury and inverse for lead) 49,68 .

There is some scientific debate about mercury effect on lymphocytes. It is suggested that the difference observed (lymphopenia x lymphocytosis) could be explained by mercury's level and form, in a way those exposed to methylmercury would be prone to lymphopenia and those to metallic or inorganic mercury to lymphocytosis 50,52,69,70. The latter effect on lymphocytes was observed in six out of seven studies, which might corroborate this theory.

Some hematological effects are considered quite severe according to preestablished criteria and can lead to a number of critical clinical conditions. They were seen at this review as a consequence of mercury's direct effect on blood cell and their corresponding clinical pictures, mainly as severe bleeding, but also as renal insufficiency, multiple organ dysfunction syndrome and sepsis. Despite the reports of other potential severe hematological effects, such as polycythemia, thrombocytosis and lymphocytosis, there were no cases of thrombosis or hematological cancer.

Mercury is a toxic substance that can lead to death. Its lethal dose is defined at 150 to $300 \mathrm{mg} /$ $70 \mathrm{~kg}$ 6. There were 29 reports of it mainly on non-occupational exposure, especially due to use of medicine in a chronic way. In the past it was prescribed as laxative, diuretic and antiseptic. Nowadays, mercury is still present in some traditional therapies and religious practices (e.g. Santería, Espiritismo or Ayurvedic medicine) 71,72 as well as in vaccine preservative. All the five occupational deaths were related to higher level of mercury exposure at acute setting.

There are some explanations for some mercury hematological effects, such as: pancytopenia due to direct toxic effect on bone marrow 11,67; anemia due to apoptosis 14,15, loss of blood from direct effect on gastrointestinal mucosa 73 and hemolysis 14,15,36; polycythemia from increased level of erythropoietin 45,74; leukopenia, neutropenia, lymphopenia and basopenia due to passed inflammatory reaction 46 and apoptosis 69,70; leukocytosis and neutrophilia due to lung inflammatory reaction (pneumonitis) 75,76 ; eosinophilia related to hypersensitivity 77,78 and idiosyncrasy 79 ; lymphocytosis due to increased calcium content in cytoplasm 23 , and; thrombocytopenia immunologically mediated 39,80 .

The actual dimension of mercury's hematologic effects is unknown for many reasons that come from the lack of studies that could evaluate this topic as a primary goal, which was discussed by two studies 39,81 to the lack of knowledge of mercury role on this subject. In the latter, two situations were observed: the physician did not request mercury biomarker when evaluating an hematological effect or he did not request blood cell count when evaluating a case of mercury intoxication, merely because of the lack of knowledge. In this review, only 14 studies had hematological effect as main outcome of mercury exposure and 381 out of 1,158 studies were excluded due to the fact of not requesting blood cell count.

A meta-analysis was not pursued because the only hematological effect that had a sufficient number of comparable groups and a statistical measure (correlation coefficient $r$ ) was lymphocytes 
alteration (lymphocytosis or lymphopenia). There were differences in reporting this measure: one study did not report the $r$ value and four, its statistical significance.

We have tried to mitigate the publication bias by a comprehensive, sensitive, unrestricted search for language, with a long period of time (more than 70 years) and search in the gray literature (e.g. congress, master and thesis). We were able to retrieve a significant amount of normal blood cell count results between exposed people as a consequence of a more sensitive search that included blood cell count as a key term. As a meta-analysis was not done, both the visual evaluation of the funnel plot and the statistical tests of hypothesis were not performed.

The quality assessment of these studies was considered weak according to EPHPP, a quality assessment tool (global ratings: 3 ) for 75 studies out of 80 . This poor quality of most studies, mainly due to the study design, absence of possible confounders' evaluation and presence of bias risk, limited the power of the epidemiological studies included. However, these data were able to identify, in absolute terms, $20 \%$ of hematological effects on the presence of mercury exposure, in particular for anemia, lymphopenia, neutrophilia and basophilia, as statistical tools with significant p-value were used. Without any doubt, this should stimulate further researches with special attention to studies of methodological elaboration. All steps of this process must be thoroughly thought, including random selection, comparison between exposure and non-exposure groups, control for confounders according to bone marrow cell affected (e.g. micronutrients, enteroparasitosis, malaria, others infections, glutathione $\mathrm{S}$ transferase deletion polymorphisms) and data collection methods that should be reliable and valid. For obvious ethical reasons, no clinical trials will be conducted to study this potential association. However, there are some others observational studies that can be done aiming, for example the frequency of this outcome (cross-sectional with comparing groups; case-control; multicentric cohort studies), the risk assessment of this exposure, as well to ascertain the clinical significance of this relationship.

\section{Conclusion}

This review was able to retrieve a significant number of studies for an issue with sparse information, although only few of them have evaluated hematological effect as the primary outcome. Despite the fact that the majority of exposed individuals had normal blood cell count and mercury hematological effects do not seem very usual, few studies reported association from refined observational study designs including robust statistical analysis, especially for anemia, lymphopenia, neutrophilia and basophilia. In this way, the effects of mercury on health should receive worldwide attention because of its toxicity and wide source of human exposure. Researchers, as well as health practitioners, should be aware of the potential hematological effect as sometimes it can be severe and even lethal. 


\section{Contributors}

A. S. Vianna, E. P. Matos, and C. I. R. F. Asmus contributed in the elaboration of study and search terms, assessment of the articles reviewed, and article writing. I. M. Jesus contributed in the elaboration of study and article writing. V. M. Câmara contributed in the elaboration of study and search terms, discussion of results, and article writing.

\section{Additional informations}

ORCID: Angélica dos Santos Vianna (0000-00030657-2141); Elisabete Pedra de Matos (0000-00024027-6058); Iracina Maura de Jesus (0000-00019828-3720); Carmen Ildes Rodrigues Fróes Asmus (0000-0002-9864-6656); Volney de Magalhães Câmara (0000-0002-6596-6653).

\section{Acknowledgments}

We thank Roberto J. G. Unger (Public Health Institute, Federal University of Rio de Janeiro) and Prof. Eliane Pedra Dias (School of Medicine, Fluminense Federal University) for their assistance in the article review.

\section{References}

1. Contreras C, Vásquez N, Díaz L. Intoxicación acidental con mercúrio elemental. Acta Med Per 2017; 30:26-30.

2. United Nations Environment Programme. The global mercury modelling: update of modelling results in the Global Mercury Assess ment 2013. Geneva: United Nations Environment Programme; 2015.

3. Tchounwou PB, Yedjou CG, Patlolla AK, Sutton DJ. Heavy metals toxicity and the environment. EXS 2012; 101:133-64.

4. Clarkson TW. The three modern faces of mercury. Environ Health Perspect 2002; 110 Suppl 1:11-23.

5. Esdaile LJ, Chalker JM. The mercury problem in artisanal and small-scale gold mining. Chemistry 2018; 24:6905-16.

6. Hong YS, Kim YM, Lee KE. Methylmercury exposure and health effects. J Prev Med Public Health 2012; 45:353-63.

7. Hacon S, Barrocas PRG, Vasconcellos ACS, Barcellos C, Wasserman JC, Campos RC, et al. An overview of mercury contamination research in the Amazon basin with emphasis on Brazil. Cad Saúde Pública 2008; 24:1479-92.

8. Bernhoft RA. Mercury toxicity and treatment: a review of the literature. J Environ Public Health 2012; 2012:460508.

9. Rice KM, Walker EM, Wu M, Gillette C, Blough ER. Environmental mercury and its toxic effects. J Prev Med Public Health 2014; 47:74-83.

10. McHale CM, Zhang L, Smith MT. Current understanding of the mechanism of benzeneinduced leukemia in humans: implications for risk assessment. Carcinogenesis 2012; 33:24052.

11. Priya N, Nagaprabhu VN, Kurian G, Seethalakshmi N, Rao GG, Unni VN. Aplastic anemia and membranous nephropathy induced by intravenous mercury. Indian J Nephrol 2012; 22:451-4.

12. Queiroz MLS, Dantas DCM. B lymphocytes in mercury-exposed workers. Pharmacol Toxicol 1997; 81:130-3.

13. Zavariz C, Glina DMR. Efeitos da exposição ocupacional ao mercúrio em trabalhadores de uma indústria de lâmpadas elétricas localizada em Santo Amaro, São Paulo, Brasil. Cad Saúde Pública 1993; 9:117-29.

14. Zolla L, Lupidib GL, Bellellic A, Amiconicet G. Effect of mercuric ions on human erythrocytes. Relationships between hypotonic swelling and cell aggregation. Biochim Biophys Acta 1997; 1328:273-80.

15. Suwalsky M, Ungerer B, Villena F, Cuevas F, Sotomayor CP. $\mathrm{HgCl}^{2}$ disrupts the structure of the human erythrocyte membrane and model phospholipid bilayers. J Inorg Biochem 2000; 81:267-73. 
16. Deem SL, Norton TM, Mitchell M, Segars A, Alleman AR, Cray C, et al. Comparison of blood values in foraging, nesting, and stranded loggerhead turtles (Caretta caretta) along the coast of Georgia, USA. J Wildl Dis 2009; 45:41-56.

17. Seriani R, França JG, Lombardi JV, Brito JM, Ranzani-Paiva MJ. Hematological changes and cytogenotoxicity in the tilapia Oreochromis niloticus caused by sub-chronic exposures to mercury and selenium. Fish Physiol Biochem 2015; 41:311-22.

18. Liberati A, Altman DG, Tetzlaff J, Mulrow C, Gøtzsche PC, Ioannidis JP, et al. The PRISMA statement for reporting systematic reviews and meta-analyses of studies that evaluate health care interventions: explanation and elaboration. J Clin Epidemiol 2009; 62:e1-e34.

19. Galvão TF, Pereira GM. Revisões sistemáticas da literatura: passos para sua elaboração. Epidemiol Serv Saúde 2014; 23:183-4.

20. Bauer KA. Maternal adaptations to pregnancy: hematologic changes. UpToDate 2018; 27 apr. https://www.uptodate.com/contents/ maternal-adaptations-to-pregnancy-hemato logic-changes (accessed on 28/Apr/2018).

21. Effective Public Health Practice Project. Quality assessment tool for quantitative studies. https://merst.ca/ephpp/ (accessed on 21/ Apr/2018)

22. Moszczyński P, Slowiński S. The behaviour of T-cell subpopulations in the blood of workers exposed to mercury. Med Lav 1994; 85:23941.

23. Moszczyński P, Słowiński S, Rutkowski J, Bem S, Jakus-Stoga D. Lymphocytes, T and NK cells, in men occupationally exposed to mercury vapours. Int J Occup Med Environ Health $1995 ; 8: 49-56$

24. Soleo L, Colosio C, Alinovi R, Guarnieri D, Russo A, Lovreglio F, et al. Immunological effects of exposure to low exposure to inorganic mercury. Med Lav 2002; 93:225-32.

25. Lauwerys R, Bonnier C, Evrad P, Gennart JP, Bernard A. Prenatal and early postnatal intoxication by inorganic mercury resulting from the maternal use of mercury containing soap. Hum Toxicol 1987; 6:253-6.

26. Montoya-Cabrera MA, Rubio-Rodríguez S, Velázquez-González E, Avila SM. Intoxicación mercurial causada por un medicamento homeopático. Gac Méd Méx 1991; 127:260-70.

27. Bender CE, Hoxsey RJ, DeMarsh QB. Neutropenia in a patient treated with a mercurial diuretic and its response to BAL. Ann Intern Med 1950; 33:1285-90.

28. Portwich F, Maron H. Fatal allergic thrombopenia after influenza prophylaxis with phenylmercuric borate (merfen). Arztl Wochensch 1959; 14:65-6.
29. Wilson DR. Mercurial poisoning and aplastic anaemia. BMJ 1966; 2:1534.

30. Johnson KG, Evanger A, van Meter W. Elemental mercury poisoning manifest by acrodynia and neutropenia. Vet Human Toxicol 1978; 20:404-9.

31. Murphy MJ, Culliford EJ, Parsons V. A case of poisoning with mercuric chloride. Resuscitation 1979; 7:35-44.

32. Slee PH, den Ottolander GJ, de Wolff FA. A case of merbromin (mercurochrome) intoxication possibly resulting in aplastic anemia. Acta Med Scand 1979; 205:463-6.

33. Pavithran K. Mercury and aplastic anemia. Natl Med J India 1994; 7:252.

34. Alvarado EV, Ramírez JA, Nilsman M, Bonilla R. Intoxicación sistemática por mercurio elemental depositado en el organismo. Medicina Legal 1995; 12:36-40.

35. Dada MA, Jogessar V, Ramdial PK. Membranous fat necrosis and aplastic anaemia due to mercury poisoning. J Pathol 1999; 189 Suppl $\mathrm{S}: 22 \mathrm{~A}$.

36. De Palma G, Mariotti O, Lonati D, Goldoni M, Catalani S, Mutti A, et al. Toxicokinetics and toxicodynamics of elemental mercury following self-administration. Clin Toxicol (Phila) 2008; 46:869-76.

37. Yildrim R, Erdem F, Gündogdu M, Bilen Y, Koca E, Yillikoglu Y, et al. Mercury toxicity: a family case report. Turk J Hematol 2012; 29:76-9.

38. Ryrie DR, Toghill PJ, Tanna MK, Galan GN. Marrow suppression from mercury poisoning? BMJ 1970; 1:499.

39. Fuortes LJ, Weismann DN, Graeff ML, Bale JF, Tannous R, Peters C. Immune thrombocytopenia and elemental mercury poisoning. Clin Toxicol (Phila) 1995; 33:449-55.

40. Erkek N, Senel S, Sarac A, Ertan U, Karacan CD. Being alive after a severe inorganic mercury intoxication. Eur J Pediatr 2010; 1 69:625-8.

41. Butt EM, Simonsen DG. Mercury and lead storage in human tissue. Am J Clin Pathol 1950; 20:716-23.

42. Oluwole AF, Asubiojo OI, Adekile AD, Filby RH, Bragg A, Grimm CI. Trace element distribution in the hair of some sickle cell anemia patients and controls. Biol Trace Elem Res 1990; 26-27:479-84.

43. Siblerud RL. The relationship between mercury from dental amalgam and the cardiovascular system. Sci Total Environ 1990; 99:23-35.

44. Langworth S, Elinder EG, Sundqvist KG. Minor effects of low exposure to inorganic mercury on the human immune system. Scand J Work Environ Health 1993; 19:405-13. 
45. Zabiński Z, Dabrowski Z, Moszczyński P, Rutowski J. The activity of erythrocyte enzymes and basic indices of peripheral blood erythrocytes from workers chronically exposed to mercury vapours. Toxicol Ind Health 2000; 16:58-64.

46. Frisk P, Danersund A, Hudecek R, Lindh U. Changed clinical chemistry pattern in blood after removal of dental amalgam and other metal alloys supported by antioxidant therapy. Biol Trace Elem Res 2007; 120:163-70.

47. Brázdová ZD, Pomerleau J, Fiala J, Vorlová L, Müllerová D. Heavy metals in hair samples: a pilot study of anaemic children in Kazakhstan, Kyrgyzstan and Uzbekistan. Cent Eur J Public Health 2014; 22:273-6.

48. Mathee A, Naicker N, Kootbodien T, Muluma T, Nkomo P, Naik I, et al. A cross sectional analytical study of geophagia practices and blood metal concentrations in pregnant women in Johannesburg, South Africa. S Afr Med J 2014; 104:568-70.

49. Plante C, Blanchet C, Rochette L, O’Brien HT. Prevalence of anemia among inuit women in Nunavik, Canada. Int J Circumpolar Health 2011; 70:154-65.

50. Kim JH, Lee KH, Hong SC, Lee HS, Lee J, Kang JW. Association between serum mercury concentration and leukocyte differential count in children. Pediatr Hematol Oncol 2013; 32:109-14.

51. Kim KN, Bae S, Park HY, Kwon HJ, Hong YC. Low-level mercury exposure and risk of asthma in school-age children. Epidemiology 2015; 26:733-9.

52. Oulhote Y, Shamim Z, Kielsen K, Weihe P, Grandjean P, Ryder LP, et al. Children's white blood cell counts in relation to developmental exposures to methylmercury and persistente organic pollutants. Reprod Toxicol 2017; 68:207-14

53. Weinhouse C, Ortiz EJ, Berky AJ, Bullins P, Hare-Grogg J, Rogers L, et al. Hair mercury level is associated with anemia and micronutrients status in children living near artisanal ans small-scale gold mining in the Peruvian Amazon. Am J Trop Med Hyg 2017; 97:188697.

54. Douine M, Mosnier E, Le Hingrat Q, Charpentier C, Corlin F, Hureau L, et al. Illegal gold miners in French Guiana: a neglected population with poor health. BMC Public Health 2017; 18:1-20

55. Ferguson A, Penney R, Solo-Gabriele H. A review of the field on children's exposure to environmental contaminants: a risk assessment approach. Int J Environ Res Public Health 2017; 14:E265.
56. Carvalho LVB, Costa-Amaral IC, Mattos RCOC, Larentis AL. Exposição ocupacional a substâncias químicas, fatores socioeconômicos e saúde do trabalhador: uma visão integrada. Saúde Debate 2017; 41:321-6.

57. Chen CY, Driscoll CT, Eagles-Smith CA, Eckley CS, Gay DA, Hsun-Kim H, et al. A critical time for mercury science to inform global policy. Environ Sci Technol 2018; 52:9556-61.

58. Hong C, Yu X, Liu J, Chen Y, Rothenberg SE. Low-level methylmercury exposure through rice ingestion in a cohort of pregnant mothers in rural China. Environ Res 2016; 150:519-27.

59. Lee R, Middleton D, Caldwell K, Dearwent S, Jones S, Lewis B, et al. A review of events that expose children to elemental mercury in the United States. Environ Health Perspect 2009; 117:871-8.

60. Ekawanti A, Krisnayanti BD. Effect of mercury exposure on renal function and hematological parameters among artisanal and small-scale gold miners at Sekotong, West Lombok, Indonesia. J Health Pollut 2015; 5:25-32.

61. Câmara VM, Silva AP, Pivetta F, Perez MA, Lima MIM, Filhote MIF, et al. Estudo dos níveis de exposição e efeitos à saúde por mercúrio metálico em uma população urbana de Poconé, Mato Grosso, Brasil. Cad Saúde Pública 1996; 12:69-77.

62. Environmental Protective Agency. 2017 EPAFDA advice about eating fish and shellfish. https://www.epa.gov/fish-tech/2017-epafda-advice-about-eating-fish-and-shellfish (accessed on 23/Jul/2017).

63. Hacon S, Farias RA, Barrocas P, Campos RC, Wasserman J, Argento R. Avaliação da exposição humana ao mercúrio na região norte de Mato Grosso-Amazônia Legal. Cad Saúde Colet (Rio J.) 2005; 13:837-54.

64. Hacon S, Rochedo ERR, Campos RRR, Lacerda LD. Mercury exposure through fish consumption in the urban area of Alta Floresta in the Amazon Basin. J Geochem Explor 1997; 58:209-16.

65. Farias LA, Fávaro DIT, Oliveira PTMS, Braga ES. Mercury and methylmercury in the children hair and fish mostly consumed in $\mathrm{Cu}-$ batão, São Paulo State, Brazil. Rev Inst Adolfo Lutz 2014; 73:158-68.

66. Bose-O'Reilly S, McCarty KM, Steckling N, Lettmeier B. Mercury exposure and children's health. Curr Probl Pediatr Adolesc Health Care 2010; 40:186-215.

67. Winship KA. Toxicity of mercury and its inorganic salts. Adverse Drug React Acute Poisoning Rev 1985; 4:129-60. 
68. Bárány E, Bergdahl IA, Bratteby LE, Lundh T, Samuelson G, Skerfying S, et al. Iron status influences trace element levels in human blood and serum. Environ Res 2005; 98:215-23.

69. Shenker BJ, Rooney C, Vitale L, Shapiro IM. Immunotoxic effects of mercuric compounds on human lymphocytes and monocytes. I. Suppression of T-cell activation. Immunopharmacol Immunotoxicol 1992; 14:539-53.

70. Shenker BJ, Guo TL, Shapiro IM. Mercuryinduced apoptosis in human lymphoid cells: evidence that the apoptotic pathway is mercurial species dependent. Environ Res 2000; 84:89-99.

71. Masur LC. A Review of use of mercury in historic and current ritualistic and spiritual practice. Altern Med Rev 2011; 16:314-20.

72. Wu ML, Deng JF, Lin KP, Tsai WJ. Lead, mercury na arsenic poisoning due to topical use of traditional chinese medicines. Am J Med 2013; 126:451-54.

73. Doolan PD, Hess WC, Kyle LH. Acute renal insufficiency dure to biclhoride of mercury observations on gastrointestinal hemorrhage and Bal. N Engl J Med 1953; 249:273-6.

74. Campbell G, Leitch D, Lewington AJP, Dargan PI, Baker RJ. Minimal change nephrotic syndrome due to ocupational mercury vapor inhalation. Clin Nephrol 2009; 3:216-9.

75. Kanamaru M, Suzuki H, Katoh M, Nishikawa T, Ohyama Y, Noda H. Effects of agricultural chemicals on rural inhabitants in Miye prefecture: a report on the findings of health examinations. Journal of the Japanese Association of Rural Medicine 1984; 33:159-66.

76. Maramba NPC, Reyes JP, Francisco-Rivera AT, Panganiban LCR, Dioquino C, Dando N, et al. Environmental and human exposure assessment monitoring of communities near an abandoned mercury mine in the Philippines: a toxic legacy. J Environ Manage 2006; 81:135-45.

77. Tschanz C, Prins C. Drug rash with eosinophilia and systemic symptoms caused by topical application of mercury. Dermatology 2000; 201:381-2.

78. Tunnessen WW, McMahon KJ, Baser M. Acrodynia: exposure to mercury from fluorescente light bulbs. Pediatrics 1987; 79:786-9.

79. Al-Sinani S, Al-Rawas A, Dhawan A. Mercury as a cause of fulminant hepatic failure in a child: case report and literature review. Clin Res Hepatol Gastroenterol 2011; 35:580-2.

80. Gys YF, Fadeev AP. Clinical aspects of occupational intoxications by organic mercury toxic chemicals among agricultural workers in the Chelyabinsk region. Scientific Transactions of Leningrad Postgraduate Medical Institute 1971; 8:127-33.

81. Schwartz JG, Snider TE, Montiel MM. Toxicity of a family from vaccumed mercury. Am J Emerg Med 1992; 10:258-61.

82. Larsen RR, Sawyer KC, Sawyer RB, Saliba NS. Mercury granuloma complicating gastrointestinal tube decompression. Surgery 1963; 54:442-7.
83. Ross AT. Mercuric polyneuropathy with albumin-cytologic dissociation and eosinophilia. JAMA 1964; 188:830-1.

84. Hannigan BG. Self-administration of metallic mercury by intravenous injection. BMJ 1978; 2:933.

85. Wright N, Yeoman WB, Carter GF. Massive oral ingestion of elemental mercury without poisoning. Lancet 1980; 1:206.

86. Lien DC, Todoruk DN, Rajani HR, Cook DA, Herbert FA. Accidental inhalation of mercury vapour: respiratory and toxicologic consenquences. Can Med Assoc J 1983; 129:591-5.

87. McNeil NI, Issler HC, Olver RE, Wrong OM. Domestic metallic mercury poisoning. Lancet 1984; 1:269-71.

88. Foulds DM, Copeland KC, Franks RC. Mercury poisoning and acrodynia. Am J Dis Child 1987; 141:124-5

89. Oliveira DB, Foster G, Savill J, Syme PD, Taylor A. Membranous nephropathy caused by mercury containing skin lightening cream. Postgrad Med J 1987; 63:303-4.

90. Murray KM, Hedgepeth JC. Intravenous selfadministration of elemental mercury: efficacy of dimercaprol therapy. Drug Intell Clin Pharm 1988; 22:972-5.

91. Dell'Omo M, Muzi G, Bernard A, Filiberto S, Lauwerys RR, Abbritti G. Long term pulmonar and systemic toxicity following intravenous mercury injection. Arch Toxicol 1997; 72:59-62.

92. Chodorowski Z, Anand JS. Intravenous selfadministration of metallic mercury by two alcohol abusers. Przegl Lek 2000; 57:585-7.

93. González R, Rodulfo A, Botero M. Púrpura de Henoch-Schönlein secundaria a hipersensibilidad por mercurio en un preescolar: a propósito de un caso. Arch Venez Pueric Pediatr 2001; 64:156-62.

94. Deschamps F, Strady C, Deslee G, MenciereFaroy B, Deschamps S. Five years of follow-up after elemental mercury self-poisoning. Am J Forensic Med Pathol 2002; 23:170-2.

95. Langworth S, Björkman L, Elinder CG, Järup L, Savlin P. Multidisciplinary examination of patitens with illness attributes to dental fillings. J Oral Rehabil 2002; 29:705-13.

96. Winkler R, Schaffer AW, Komaris C, Barth A, Giovanoli P, Osterode W, et al. Health consequences of intravenous injection of metallic mercury. Int Arch Occup Environ Health 2002; 75:581-6.

97. Koyun M, Akman S, Güven AG. Mercury intoxication resulting from school barometers in three unrelated adolescents. Eur J Pediatr 2004; 163:131-4.

98. Glezos JD, Albrecht JE, Gair RD. Pneumonitis after inhalation of mercury vapours. Can Respir J 2006; 13:150-2.

99. Matushita JPK, Peixoto RMA, Leão Filho HM, Missiaggia GC, Dias RS, Tavares Junior WC, et al. Embolismo por mercúrio metálico: relato de caso. Radiol Bras 2007; 40:217-9. 
100. Bamonti F, Guzzi G, Ferrero E. Cracked mercury dental amalgam as possible cause of fever of unknown origin: a case report. J Med Case Rep 2008; 2:72.

101. Girault PY, Rey P, Veran Y. Acute hepatitis after cutaneous application of an artisanal topical ointment with mercury. Gastroenterol Clin Biol 2008; 32:819-23.

102. Berrouet Mejía MC, Escobar Toledo IE, González Ramírez DM. Toxicidad sistémica asociada con la inyección intramuscular de mercúrio metálico: reporte de un caso. Iatreia 2008; 21:420-5.

103. Sarikaya S, Karcioglu O, Ay D, Cetin A, Aktas $\mathrm{C}$, Serinken M. Acute poisoning: a case report. BMC Emerg Med 2010; 10:7.

104. Khoury EDT, Souza GS, Silveira LCL, Costa CA, Araújo AA, Pinheiro MCN. Manifestações neurológicas em ribeirinhos de áreas expostas ao mercúrio na Amazônia brasileira. Cad Saúde Pública 2013; 29:2307-18.

105. Beasley DM, Schep LJ, Slaughter RJ, Temple WA, Michel JM. Full recovery from a potentially lethal dose of mercuric chloride. J Med Toxicol 2014; 10:40-4.

106. Dardamanis M, Balta L, Zacharopoulos V, Tatsi V, Tzima H. An unexpected foreign body (a thermometer) in the bladder: a case report. Urol Case Rep 2014; 2:65-6.

107. Cicek-Senturk G, Altay FA, Ulu-Klilic A, Gurbuz Y, Tutuncu E, Sencan I. Acute mercury poisoning presenting as fever of unknown origin in an adult woman: a case report. J Med Case Rep 2014; 8:266.
108. Devlin HH, Sudlow M. Peripheral mercury embolization ocurring during arterial blood sampling. BMJ 1967; 1:347-8.

109. Takamatsu M, Maeda H, Inuzuka T, Futatsuka M, Arimatsu Y. Health hazards of workers engaged in the laboratory of a fungicide factory. Journal of the Kumamoto Medical Society 1969; 43:93-8.

110. Nizov AA, Shestakov HM. Contribution to the clinical aspects of granosan poisoning. Sov Med 1971; 11:150-2.

111.Jung RC, Aaronson J. Death following inhalation of mercury vapor at home. West J Med 1980; 132:539-43.

112. Torresani C, Caprari E, Manara GC. Contact urticaria syndrome due to phenylmercuric acetate. Contact Dermatitis 1993; 29:282-3.

113. Melo B, Cortes B, Mujica G, Acosta M, Cortez A, Bourg D, et al. Exposición mercurial y estado de salud del personal que labora en el servicio de odontología del IPASME Barquisímeto. Acta Odontol Venez 2000; 38:24-31.

114. Rodríguez EA, Tubio FPM, Ferrera JMD. Enfermedades de los trabajadores de las minas de Almadén y Almadenejos (España, 1883-1887). Salud Trabaj (Maracay) 2011; 19:147-59.

115. Alhamad T, Rooney J, Nwosu A, Maccombs J, Kim YS, Shukla V. Lessons learned from a fatal case of mercury intoxication. Int Urol Nephrol 2012; 44:647-51. 


\section{Resumo}

O mercúrio é um metal que pode ser encontrado naturalmente no meio ambiente e através de fontes antropogênicas. É altamente tóxico para ecossistemas e seres vivos. A maior parte da exposição humana provém da ingestão de pescados contaminados, da liberação de gases da amálgama dentária ou da exposição ocupacional (p.ex.: extração de ouro). Vastas populações são expostas ao mercúrio, tornando-se uma questão de saúde pública muito importante. Efeitos adversos à saúde são comumente observados no sistema nervoso, mas todos os órgãos são alvos em potencial, como a medula óssea. O principal objetivo do estudo foi avaliar as evidências disponíveis sobre a exposição humana ao mercúrio e seus efeitos hematológicos. Uma estratégia de busca foi realizada, incluindo termos chave (palavras-chave, palavras do texto e equivalentes), para pesquisar dois repositórios de dissertações de mestrado e teses de doutorado (Fiocruz/ARCA e Universidade de São Paulo) e quatro bases de dados eletrônicas: BVS/LILACS, MEDLINE/PubMed, Scopus e TOXLINE/NIH (artigos publicados de 1950 até fevereiro de 2018). Não houve restrições de linguagem e uma ferramenta (EPHPP) foi utilizada para avaliar a qualidade dos estudos incluidos. De acordo com os critérios pré-estabelecidos, foram encontrados 80 estudos, todos observacionais (48 relatos de caso, 24 estudos transversais, 6 séries de casos e 2 coortes), que compreendiam 9.284 pessoas. Apesar do fato de que as pessoas mais expostas (6.012) tinham contagens de células sanguíneas normais, $e$ os efeitos hematológicos do mercúrio não pareciam muito comuns (1.914 casos, 14 graves e 29 mortes), três estudos relataram a associação de $(\beta)$ anemia, linfopenia, neutrofilia e basofilia. Concluímos que as informações coletadas indicam efeitos hematotóxicos do mercúrio, alguns dos quais podem ser muito graves e até fatais.

Intoxicação por Mercúrio; Intoxicação por Metais Pesados; Mercúrio; Contagem de Células

Sanguíneas

\section{Resumen}

El mercurio es un metal que se puede encontrar de forma natural en el ambiente y mediante fuentes antropogénicas. Es altamente tóxico para los ecosistemas y seres vivos. Entre otras, la mayor parte de la exposición humana, proviene de la ingestión de pescado contaminado, liberación de gases de amalgamas dentales o exposición ocupacional (p.ej. extracción de oro). Vastas poblaciones están expuestas al mercurio, convirtiéndolo en un asunto muy importante desde la perspectiva de la salud pública. Los efectos adversos para la salud se observan comúnmente en el sistema nervioso, pero cada órgano es un objetivo potencial, como la médula ósea. El objetivo principal del estudio fue evaluar las evidencias disponibles sobre la exposición humana al mercurio y sus efectos hematológicos. Se realizó una estrategia de búsqueda, incluyendo términos clave (palabras-clave, palabras del texto $y$ equivalentes), se consultaron 2 registros de trabajos finales de máster y tesis de doctorado (Fiocruz/ARCA y Universidad de São Paulo) y 4 bases de datos electrónicas diferentes: BVS/LILACS, MEDLINE/PubMed, Scopus y TOXLINE/NIH, para artículos publicados desde el año 1950, hasta febrero de 2018. No hubo restricciones de lengua y se usó la herramienta (EPHPP) para evaluar la calidad de los estudios incluidos. De acuerdo con los criterios preestablecidos, se recopilaron 80 estudios, todos observacionales (48 informes de casos, 24 estudios transversales, 6 series de casos, $y$ 2 cohortes), que comprendieron a 9.284 personas. A pesar de que la mayoría de los expuestos (6.012) tenian un recuento normal de células sanguíneas y los efectos hematológicos del mercurio no parecían muy comunes (1.914 casos: 14 severos y 29 muer tes), tres estudios informaron de la asociación $(\beta)$ para anemia, linfopenia, neutrofilia y basofilia. Concluimos que la información recabada indicaba los efectos hematotóxicos del mercurio, algunos de los cuales pueden ser muy serios e incluso fatales.

Intoxicación por Mercurio; Intoxicación por

Metales Pesados; Mercurio; Recuento de

Células Sanguíneas
Submitted on 11/May/2018

Final version resubmitted on 16/Oct/2018

Approved on 25/Oct/2018 